\title{
SCIENTIFIC REPORTS

\section{Global Prevalence and Risk Factors of Gastro-oesophageal Reflux Disease (GORD): Systematic Review with Meta-analysis}

\author{
Jorabar Singh Nirwan, Syed Shahzad Hasan (D), Zaheer-Ud-Din Babar, Barbara R. Conway (iD) \& \\ Muhammad Usman Ghori $\mathbb{1}^{*}$
}

\begin{abstract}
Although gastro-oesophageal reflux disease (GORD) is a common medical complaint, there is currently no consensus on the global prevalence of GORD. The aim of this study was to conduct a systematic review and meta-analysis on GORD prevalence and risk factors at a global level. MEDLINE, EMBASE, CINAHL, Scopus, Cochrane library, and Google Scholar were systematically searched, without language restrictions, for studies on the prevalence and risk factors of GORD. Data were pooled using a random effects model ( $95 \%$ confidence interval), and the odds ratio and relative risk for each risk factor were calculated. Out of 34,355 search results, 96 records reporting the results from 102 studies fulfilled the inclusion criteria, representing 37 countries and all regions of the UN geoscheme. The global pooled prevalence of GORD was $13.98 \%$ and varied greatly according to region (12.88\% in Latin America and the Caribbean to $19.55 \%$ in North America) and country ( $4.16 \%$ in China to $22.40 \%$ in Turkey). Using the United Nations 2017 Revision of World Population Prospects, the estimated number of individuals suffering from GORD globally is 1.03 billion. Multiple risk factors associated with a significant increase in the risk of GORD were also identified. This systematic review and meta-analysis revealed that although a substantial proportion (13.98\%) of the global population suffers from GORD, there are significant variations between regions and countries. Risk factors for GORD were also identified which may allow clinicians to recognise individuals most at risk.
\end{abstract}

Gastro-oesophageal reflux disease (GORD) is one of the most common complaints in general medical practice and can be a debilitating condition requiring life-long medication, invasive surgery, and lifestyle changes ${ }^{1,2}$. It is widely accepted that the pathophysiology of GORD is multifactorial representing different ends of a spectrum varying with the severity of reflux rather than distinct pathophysiological mechanisms ${ }^{3}$. A number of factors have been suggested to cause GORD including an increased compliance of the oesophagogastric junction (OGJ), and a higher pressure gradient across the $\mathrm{OGJ}{ }^{4}$. Additionally, differences in the meal distribution or the localisation of the acid pocket on top of the meal, as well as a hypotensive lower oesophageal sphincter (LOS), and a defective gastric sling/clasp muscle fibre component, may also lead to the occurrence of GORD symptoms ${ }^{5,6}$.

In addition to having significant undesirable effects on an individual's health-related quality of life, GORD has also shown to have a significant economic and societal burden. Although recent studies regarding the economic evaluation of GORD are scarce, previous studies have estimated that the resource implications of GORD are approximately $£ 760$ million/year in the UK, whereas in the USA, the cost of health care and lost productivity due to GORD are an estimated $\$ 24$ billion/year ${ }^{7-11}$. Furthermore, it has been estimated that the total direct costs (physician visit, costs of drugs, costs of tests, and hospitalisation) and indirect costs (number of days with total productivity loss and number of days with at least 30\% lower functionality) of GORD per patient in Iran during 6 months are $\$ 97.90$ purchasing power parity dollars (PPP) and $\$ 13.70$ PPP respectively ${ }^{12}$. Overall, these statistics highlight the need for this condition to receive more global awareness.

Although a number of systematic reviews investigating the prevalence of GORD according to specific regions or countries have been conducted, evaluations of epidemiological studies from around the world are limited and have been a challenge for investigators due to language and cultural differences in symptom interpretation ${ }^{13-17}$. 
A recent systematic review and meta-analysis conducted by Eusebi et al. 2017 included 108 studies and assessed the global prevalence of, and risk factors for, gastric reflux symptoms rather the prevalence of GORD ${ }^{18}$. The aim of this review, to evaluate the global prevalence of reflux symptoms, was met. However, as these symptoms may be an indication of other gastric conditions such as irritable bowel syndrome, the global prevalence of GORD was not estimated.

More relevantly to the current review, in 2005, Dent et al. conducted a systematic review to evaluate the global prevalence of GORD using stringent selection criteria and a GORD definition of at least weekly heartburn and/ or acid regurgitation, or diagnosed by a physician ${ }^{19}$. This review was updated by El-Serag et al. in 2013 and, in total, only included 28 studies $^{20}$. Furthermore, their review excluded studies not published in English and did not identify any studies from Africa. Risk factors for GORD were also not thoroughly explored; however, the authors stated that this was not the primary goal of their review. Hence, to the best of our knowledge, the current literature is missing a recent comprehensive global systematic review on the prevalence of GORD with associated meta-analyses since the review conducted by El-Serag et al. in 2013, and devoid of an extensive global scale systematic review on the risk factors for GORD. Therefore, the aim of the current review was to search the literature systematically using PRISMA (Preferred Reporting Items for Systematic Reviews and Meta-Analyses) 2009 guidelines and estimate the global prevalence of GORD, the prevalence of GORD according to geographical location and to identify risk factors associated with an increased risk of the condition ${ }^{21}$. It is predictable that being aware of the demographics of GORD patients and the risk factors for GORD permits clinicians to identify individuals most at risk, allowing early diagnosis and commencement of treatment, as well as highlighting areas which require more attention from researchers and clinicians.

\section{Methods}

Scope of review: eligibility criteria. This systematic review was performed in accordance with the PRISMA 2009 guidelines ${ }^{21}$. The primary investigators (JSN and MUG) screened titles and abstracts for articles reporting (a) prevalence of GORD, (b) risk factors associated with GORD, and (c) regional differences in the prevalence of GORD. Studies focusing on pathophysiology, lifestyle approaches or interventions, and evaluations of clinical data were excluded, whereas original studies (e.g. longitudinal studies, cross-sectional studies) on prevalence of GORD were included. Meta-analyses were conducted where $\geq 4$ studies were available. The publication period was from $1^{\text {st }}$ January 1947 to $30^{\text {th }}$ June 2018 and included studies on subjects of any age group, with no language restrictions applied as studies not published in English were translated using an in-kind support from native speakers.

Information sources. The following databases were searched: MEDLINE, EMBASE, Cumulative Index to Nursing and Allied Health Literature (CINAHL), Scopus, Cochrane library, and Google Scholar with the last update on $30^{\text {th }}$ June, 2018. Reference lists of articles identified in the search and relevant review articles were included and were subject to the same eligibility evaluation.

Searching. The search strategy identified research on prevalence and risk factors of GORD. Search terms were 'gastroesophageal reflux', 'GERD', 'GORD', 'heartburn', 'esophagitis' or 'oesophagitis' combined with 'epidemiology', 'epidemiological', 'prevalence, 'incidence' or 'population' in the title, abstract or list of medical subject heading terms. Titles and abstracts were screened to remove studies that were evidently irrelevant to the aim of the review. Systematic reviews, meta-analyses, conference presentations, and letters or correspondences were all excluded from this review. The full texts of the remaining studies were then examined to determine eligibility.

Study selection. Two investigators (JSN and MUG) assessed abstracts independently against the following criteria: (1) studies assessing prevalence with or without risk factors of GORD; (2) original studies (longitudinal and cross-sectional) studies; (3) inclusion of any age group; and (4) studies which recruited more than 50 participants. Full papers of potential studies were independently assessed by the two investigators for their suitability.

Data extraction process. Studies were catalogued according to the selected criteria and data was extracted to a Microsoft Excel ${ }^{\circledR} 2017$ spreadsheet. Data extracted from eligible studies included: response rate, continent, country and geographical location of study, sample size, age range of sample population, sample size according to age group, sample size according to gender, method of data collection, criteria used to define GORD, instrument used to collect data, duration of symptoms, overall prevalence of GORD, and prevalence of GORD according to risk factors.

Data Items/study characteristics. Prevalence of GORD. GORD was defined as one or more of the following: heartburn and/or acid regurgitation at least once a week regardless of severity of symptoms, diagnosed by a clinician, according to a score-based GORD specific questionnaire, or the Montreal definition of mild symptoms occurring at least twice a week or moderate to severe symptoms occurring at least once a week. Studies reporting at least weekly prevalence of only one GORD symptom were excluded to avoid bias in the results. Study populations were required to be representative of the general population, therefore, those studies which reported the prevalence of GORD in sub-groups, such as hospital patients, employees at an institution, or patients suffering from a particular disease, were excluded.

Risk factors of GORD. All eligible articles were screened to identify studies that investigated any risk factors of GORD. The risk factors assessed in this review include: gender, age group, self-reported alcohol intake, BMI level, education level, marriage status, self-reported NSAIDs/aspirin use, area of domicile, self-reported smoking habits, income-level, and diet (spicy food, sweet food, meat/fish, carbonated drinks, fatty food, fried food, and coffee/ tea). Low education level was defined as uneducated, primary school level only, less than high school, or 0-8 years 


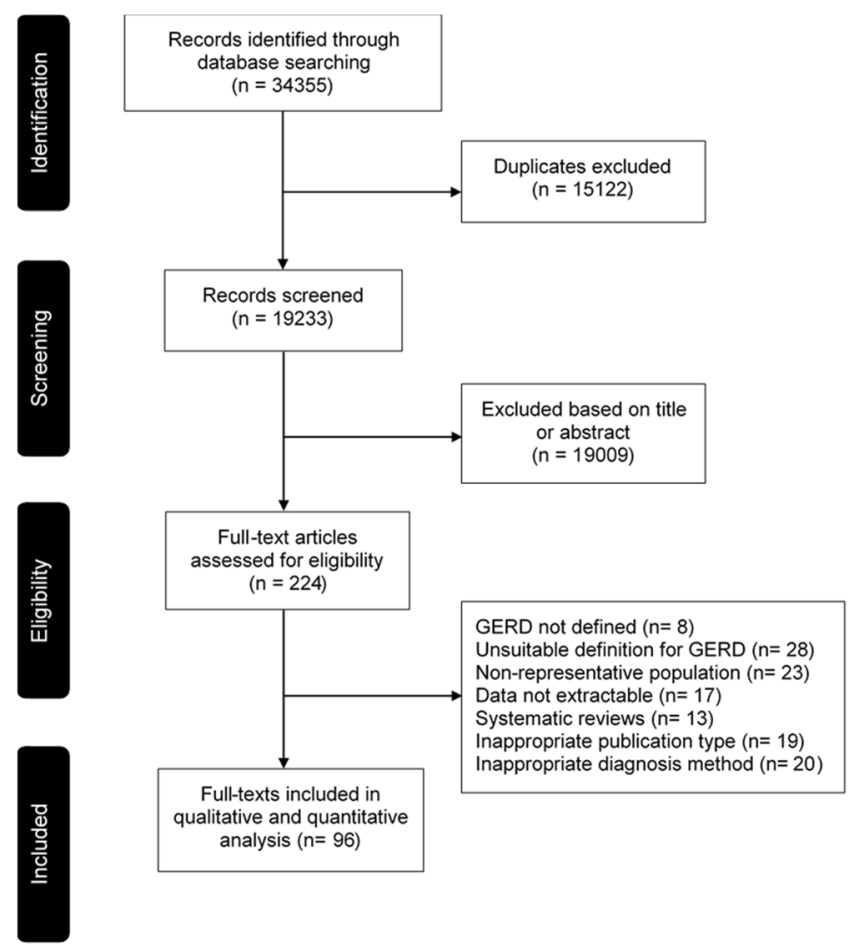

Figure 1. Flow-diagram of study selection.

of study; medium education level was defined as up to secondary school/high school or 9-12 years of study; and high education level was defined as college/university or $\geq 13$ years of study. Income-level was categorised as low, medium, or high according to the criteria used by the respective study. Data regarding the prevalence of GORD according to exposure and non-exposure of risk factors was then extracted.

Quality assessment. The quality of the papers included was rated according to the Newcastle-Ottawa Quality Assessment Scale adapted for cross-sectional studies ${ }^{22}$. The quality assessment results are shown in Table S1.

Statistical analysis. All statistical analyses, apart from odds ratio (OR) and risk factor (RR), were conducted using MetaXL version 5.3. OR and RR were calculated using MedCalc online calculators ${ }^{23,24}$. The prevalence of GORD in each study was pooled using a random effects model to give an estimate of the global prevalence of GORD. Heterogeneity across studies was assessed using the $\mathrm{I}^{2}$ statistic with a cut off of $50 \%$ and the $\chi^{2}$ test with a $P$ value of $<0.10$ as the threshold for statistically significant heterogeneity.

The prevalence of GORD according to the geographical location was grouped according to the United Nations (UN) geoscheme ${ }^{25}$. Population data for regions and countries was obtained from the UN 2017 Revision of World Population Prospects ${ }^{26}$, and the number of people suffering from GORD in a specific geographical location was calculated by extrapolating the pooled prevalence of GORD to the respective population data. Potential sources of heterogeneity were investigated by stratifying pooled prevalence of GORD by (a) criteria used to define GORD, (b) duration of symptoms, (c) instrument used for data collection and (d) method of data collection. The effect of risk factors on the prevalence of GORD was investigated by calculating OR and RR of risk factors, with a 95\% confidence interval (CI).

\section{Results}

Search results. A total of 34,355 records were retrieved from the databases after implementing the search strategy. Of these, 15,122 duplicates were excluded as well as 19,009 records excluded based on titles or abstracts. Therefore, 224 records were eligible for full-text review, of which 96 records reporting the results from 102 studies fulfilled the inclusion criteria representing 37 countries and included 469,899 participants (Fig. 1) 27-122 $^{\text {. The }}$ main reasons for exclusion were the use of an unsuitable definition for GORD (28 studies) and the inclusion of a non-representative population (23 studies). 5 studies were translated into English using an in-kind support of native speakers ( 2 from Persian and 1 each from Russian, Spanish and French). The details of the included studies are provided in Table S2.

Prevalence of GORD according to geographical location. From the 102 included studies (96 records), 63,394 subjects were diagnosed with GORD giving an overall pooled prevalence of $13.98 \%$ (95\% CI $12.47 \%-$ 15.56\%). Using the UN 2017 Revision of World Population Prospects, the estimated number of individuals suffering from GORD globally is 1.03 billion. A funnel plot representing all of the studies included in this systematic 
review is depicted in Fig. S1 and also showed significant publication bias. Forest plots of the pooled prevalence of GORD stratified by country and UN region are shown in Figs. S2-S16. The distribution of the prevalence of GORD according to geographical location is shown in Table 1 and is visually depicted in Figs. 2-4. Upon visual analysis, Fig. 2 reveals substantial variations in the prevalence of GORD globally with the lowest GORD prevalence being reported in a study from China (2.5\%) and the highest being reported in a study conducted in Saudi Arabia (45.4\%) (Table S2) 28,79 . This was in agreement with the findings revealed in the review conducted by El-Serag et al. who also found the highest and lowest prevalence of GORD reported in studies from Saudi Arabia and China, respectively ${ }^{20}$. However, as the data was not pooled according to country, their review did not report the overall prevalence of GORD in each country. The current review identified the countries with the highest and lowest pooled prevalence of GORD as Turkey $(22.40 \%$ (95\% CI $18.53 \%-126.53 \%)$ ), and China (4.16\% (95\% CI $3.35 \%-15.05 \%)$ ), respectively. This also highlighted the disparity in the prevalence of GORD between sub-regions within the same continent which was especially evident in Asia which contained the sub-region with the lowest GORD prevalence (East Asia) as well as the highest GORD prevalence (Middle East) (Fig. 3). Furthermore, to highlight countries with a particularly high prevalence of GORD, Fig. 4 displays the countries with a GORD prevalence greater than and less than the global pooled GORD prevalence.

Prevalence of GORD according to risk factors. Table 2 displays the prevalence of GORD according to risk factors. The OR and RR of risk factors for GORD are shown in Tables S3 and S4, respectively. Forest plots of the pooled prevalence of GORD stratified by risk factors are presented in Figs. S17-S34. The prevalence of GORD according to gender was reported in 50 studies. The pooled prevalence of GORD in females (17.17\% (95\% CI $14.22 \%-120.33 \%)$ ) was moderately higher than in males (15.69\% (95\% CI 13.15\%-118.42\%)). The OR (1.18 (95\% CI 1.15-1.20; $\mathrm{p}<0.0001)$ ) and RR $(1.15(95 \%$ CI 1.13-1.17; $<<0.0001))$ in females compared to males showed that females are slightly more at risk of suffering from GORD.

The pooled prevalence of GORD according to age groups displayed an increase with increasing age between the age groups of $18-34$ years $(8.70 \%$; $95 \%$ CI $4.39 \%-114.23 \%)$ and $35-59$ years $(14.53 \%$ (95\% CI $11.09 \%-$ $118.33 \%)$ ). However, there was a slight decrease between the age groups of $35-59$ years and $\geq 60$ years $(13.12 \%$ (95\% CI 9.59\%-117.11\%)). The OR (1.17 (95\% CI 1.11-1.24; $\mathrm{p}<0.0001)$ in those aged $35-59$ years compared with those aged $18-34$ years; $1.20(95 \%$ CI $1.12-1.28 ; \mathrm{p}<0.0001)$ in those aged $\geq 60$ years compared with those aged $18-34$ years; 1.03 (95\% CI $0.98-1.08 ; \mathrm{P}=0.2896)$ in those aged $\geq 60$ years compared with those aged $35-59$ years) was extremely modest. This was also the case in the RR $(1.15(95 \%$ CI $1.09-1.20 ; \mathrm{p}<0.0001)$ in those aged 35-59 years compared with those aged $18-34$ years; 1.17 (95\% CI 1.11-1.24) in those aged $\geq 60$ years compared with those aged $18-34$ years; $1.02(95 \%$ CI $0.98-1.07 ; \mathrm{P}=0.2892)$ in those aged $\geq 60$ years compared with those aged $35-59$ years).

The pooled prevalence of GORD according to alcohol intake showed a similar GORD prevalence in those who do not drink alcohol or have a low intake of alcohol (15.95\% (95\% CI 11.01\%-121.60\%)) compared with those who have a moderate to high intake of alcohol (15.56\% (95\% CI 11.63\%-119.93\%)). The OR and RR of GORD in those with a moderate to high intake of alcohol compared with those who do not drink alcohol or have a low intake of alcohol was also not significantly different $(\mathrm{OR}=1.07(95 \% \mathrm{CI} 1.02-1.12 ; \mathrm{P}=0.0044)$, and $\mathrm{RR}=1.06$ $(95 \%$ CI $1.02-1.11 ; \mathrm{P}=0.0044)$ ).

Stratified pooled prevalence of GORD by BMI showed an increase in GORD prevalence as BMI increased. The lowest prevalence of GORD was for those with a BMI $<18.5(6.64 \%(95 \%$ CI $3.40 \%-110.82 \%))$, whereas the highest prevalence of GORD was seen in those with a BMI $\geq 30.0(22.63 \%$ (95\% CI $17.33 \%-128.41 \%)$ ). This positive correlation between prevalence of GORD and BMI was also shown by a significant increase in OR and RR in subjects with a higher BMI compared with subjects with a lower BMI.

The pooled prevalence of GORD according to education level was also investigated. The prevalence of GORD was highest in subjects with low education level (16.78\% (95\% CI 12.32\%-121.77\%)), followed by those with medium education level (11.52\% (95\% CI 7.33\%-116.48\%)), and the lowest GORD prevalence was seen in those with a high education level (8.98\% (95\% CI 5.56\%-113.09\%)). The impact of education level on the prevalence of GORD was also shown in the OR and RR of GORD in those with low education level compared with those with medium and high education level $(\mathrm{OR}=2.11$ (95\% CI 1.99-2.24; $\mathrm{p}<0.0001)$ and 1.75 (95\% CI 1.64-1.87; $\mathrm{p}<0.0001)$, respectively; $\mathrm{RR}=1.95$ (95\% CI 1.85-2.06; $\mathrm{p}<0.0001)$ and 1.64 (95\% CI 1.55-1.74; $\mathrm{p}<0.0001)$, respectively). However, this was not supported by the OR and RR of GORD in those with medium education level compared with those with high education level $(\mathrm{OR}=0.82(95 \% \mathrm{CI} 0.77-0.89 ; \mathrm{p}<0.0001)$ and $\mathrm{RR}=0.84(95 \%$ CI 0.77-0.90; $\mathrm{p}<0.0001)$ ).

When the pooled prevalence of GORD was stratified according to marriage status, the highest prevalence of GORD was found in divorced/separated/widowed individuals (22.95\% (95\% CI 13.19\%-134.38\%)) followed by married individuals (15.98\% (95\% CI 10.48\%-122.35\%)), and the lowest GORD prevalence was seen in single individuals (12.85\% (95\% CI 6.59\%-120.71\%)). The OR and RR in divorced/separated/widowed and married individuals were also significantly greater when compared with single individuals.

The pooled prevalence of GORD according to NSAIDs/aspirin use showed a significantly greater prevalence of GORD in subjects using NSAIDs/aspirin (24.47\% (95\% CI 18.17\%-131.35\%)) compared with those who do not $(17.34 \%$ (95\% CI 13.36\%-121.72\%)). The OR and RR in those using NSAIDs/aspirin was 1.46 (95\% CI 1.33$1.60 ; \mathrm{p}<0.0001)$ and $1.36(95 \%$ CI $1.27-1.47 ; \mathrm{p}<0.0001)$, respectively.

The area of domicile also had a significant effect on the prevalence of GORD. The pooled prevalence of GORD in subjects living in an urban area was the highest (13.43\% (95\% CI 6.68\%-121.95\%)) followed by subjects living in a rural area $(11.70 \%(95 \% \mathrm{CI} 6.26 \%-118.46 \%))$. The OR and RR in subjects living in an urban area compared with those living in a rural area was $\mathrm{OR}=2.227$ (95\% CI 2.04-2.43; $\mathrm{p}<0.0001)$ and $\mathrm{RR}=2.05$ (95\% CI 1.90-2.22; $\mathrm{p}<0.0001)$ ). Only 2 studies reported the prevalence of GORD in individuals living in a suburban area, therefore, meta-analysis was not conducted on this sub-group (Table 2). 


\begin{tabular}{|c|c|c|c|c|c|c|c|c|c|c|c|c|}
\hline \multirow{2}{*}{$\begin{array}{l}\text { Geographical } \\
\text { location }\end{array}$} & \multirow{2}{*}{$\begin{array}{l}\text { No. of } \\
\text { studies }\end{array}$} & \multirow{2}{*}{$\begin{array}{l}\text { No. of } \\
\text { participants }\end{array}$} & \multicolumn{2}{|c|}{\begin{tabular}{|l} 
GORD \\
prevalence
\end{tabular}} & \multirow{2}{*}{$\begin{array}{l}\text { Total estimated } \\
\text { population } \\
\text { size }^{\mathrm{b}}\end{array}$} & \multirow{2}{*}{$\begin{array}{l}\text { Total estimated } \\
\text { prevalence of GORD, } \\
\mathbf{n}^{\mathrm{a}}(95 \% \mathrm{CI})\end{array}$} & \multicolumn{2}{|c|}{$95 \% \mathrm{CI}$} & \multirow[b]{2}{*}{$I^{2}$} & \multirow{2}{*}{$\begin{array}{l}\text { Cochran's } \\
\mathbf{Q}\end{array}$} & \multirow[b]{2}{*}{$\mathrm{Chi}^{2}, \mathbf{p}$} & \multirow[b]{2}{*}{$\mathrm{Tau}^{2}$} \\
\hline & & & $\mathrm{n}^{\mathrm{a}}$ & $\%$ & & & LCI & HCI & & & & \\
\hline \multicolumn{13}{|l|}{ Global } \\
\hline Overall & $102^{c}$ & 469899 & 63394 & 13.98 & $7,383,008,820$ & \begin{tabular}{|l|}
$1,032,144,633$ \\
$(920,661,200-$ \\
$1,148,796,172)$
\end{tabular} & 12.47 & 15.56 & 99.57 & 23218.83 & 0.010 & 0.052 \\
\hline Male & 50 & 122849 & 17763 & 15.69 & $3,724,132,110$ & \begin{tabular}{|l|}
$584,316,328$ \\
$(489,723,372-$ \\
$685,985,135)$ \\
\end{tabular} & 13.15 & 18.42 & 99.35 & 7565.56 & 0.010 & 0.067 \\
\hline Female & 50 & 138435 & 22998 & 17.17 & $3,658,876,550$ & \begin{tabular}{|l|}
$628,229,104$ \\
$(520,292,245-$ \\
$743,849,603)$ \\
\end{tabular} & 14.22 & 20.33 & 99.54 & 10671.53 & 0.010 & 0.084 \\
\hline \multicolumn{13}{|l|}{ Continents $^{b}$} \\
\hline Asia & 54 & 240451 & 32944 & 12.92 & $4,419,897,601$ & \begin{tabular}{|l|}
$571,050,770$ \\
$(464,531,238-$ \\
$686,410,097)$ \\
\end{tabular} & 10.51 & 15.53 & 99.69 & 17317.65 & 0.010 & 0.078 \\
\hline Europe & 29 & 190057 & 23833 & 14.12 & $740,813,959$ & \begin{tabular}{l|}
$104,602,931$ \\
$(89,712,570-$ \\
$120,382,268)$ \\
\end{tabular} & 12.11 & 16.25 & 99.31 & 4073.09 & 0.010 & 0.026 \\
\hline North America & 9 & 20525 & 3623 & 19.55 & $356,003,541$ & \begin{tabular}{|l|}
$69,598,692$ \\
$(55,536,552-$ \\
$84,835,644)$ \\
\end{tabular} & 15.60 & 23.83 & 97.89 & 378.91 & 0.010 & 0.024 \\
\hline $\begin{array}{l}\text { Latin America and } \\
\text { Caribbean }\end{array}$ & 4 & 12756 & 2205 & 12.88 & $416,436,111$ & \begin{tabular}{|l|}
$53,656,262$ \\
$(15,949,503-$ \\
$106,690,932)$
\end{tabular} & 3.83 & 25.62 & 99.62 & 791.78 & 0.010 & 0.102 \\
\hline Oceania & 4 & 3760 & 503 & 13.78 & $39,542,980$ & $\begin{array}{l}5,447,166(4,266,688- \\
6,746,032)\end{array}$ & 10.79 & 17.06 & 86.83 & 22.79 & 0.001 & 0.007 \\
\hline Africa $^{\mathrm{d}}$ & 2 & 2350 & 286 & $\mathrm{~N} / \mathrm{C}$ & $\mathrm{N} / \mathrm{C}$ & $\mathrm{N} / \mathrm{C}$ & $\mathrm{N} / \mathrm{C}$ & $\mathrm{N} / \mathrm{C}$ & $\mathrm{N} / \mathrm{C}$ & $\mathrm{N} / \mathrm{C}$ & $\mathrm{N} / \mathrm{C}$ & $\mathrm{N} / \mathrm{C}$ \\
\hline \multicolumn{13}{|l|}{ Countries } \\
\hline China & 10 & 36887 & 1405 & 4.16 & $1,397,028,553$ & \begin{tabular}{|l|}
$58,122,991$ \\
$(46,800,457-$ \\
$70,549,942)$
\end{tabular} & 3.35 & 5.05 & 92.95 & 127.61 & 0.010 & 0.004 \\
\hline Japan & 7 & 27912 & 4749 & 13.81 & $127,974,958$ & \begin{tabular}{|l|}
$17,673,342$ \\
$(10,135,617-$ \\
$26,810,754)$
\end{tabular} & 7.92 & 20.95 & 99.57 & 1387.52 & 0.010 & 0.063 \\
\hline South Korea & 7 & 43897 & 2206 & 5.84 & $50,593,662$ & $\begin{array}{l}2,953,899(2,266,596- \\
3,723,694)\end{array}$ & 4.48 & 7.36 & 96.65 & 179.08 & 0.010 & 0.006 \\
\hline Taiwan & 1 & 1238 & 310 & $\mathrm{~N} / \mathrm{C}$ & $\mathrm{N} / \mathrm{C}$ & N/C & $\mathrm{N} / \mathrm{C}$ & $\mathrm{N} / \mathrm{C}$ & $\mathrm{N} / \mathrm{C}$ & $\mathrm{N} / \mathrm{C}$ & $\mathrm{N} / \mathrm{C}$ & $\mathrm{N} / \mathrm{C}$ \\
\hline Indonesia & 1 & 278 & 26 & $\mathrm{~N} / \mathrm{C}$ & $\mathrm{N} / \mathrm{C}$ & $\mathrm{N} / \mathrm{C}$ & $\mathrm{N} / \mathrm{C}$ & N/C & $\mathrm{N} / \mathrm{C}$ & $\mathrm{N} / \mathrm{C}$ & $\mathrm{N} / \mathrm{C}$ & $\mathrm{N} / \mathrm{C}$ \\
\hline Bangladesh & 1 & 2000 & 110 & $\mathrm{~N} / \mathrm{C}$ & $\mathrm{N} / \mathrm{C}$ & N/C & $\mathrm{N} / \mathrm{C}$ & N/C & N/C & $\mathrm{N} / \mathrm{C}$ & $\mathrm{N} / \mathrm{C}$ & $\mathrm{N} / \mathrm{C}$ \\
\hline India & 3 & 6296 & 955 & $\mathrm{~N} / \mathrm{C}$ & $\mathrm{N} / \mathrm{C}$ & $\mathrm{N} / \mathrm{C}$ & $\mathrm{N} / \mathrm{C}$ & N/C & $\mathrm{N} / \mathrm{C}$ & $\mathrm{N} / \mathrm{C}$ & $\mathrm{N} / \mathrm{C}$ & $\mathrm{N} / \mathrm{C}$ \\
\hline Iran & 16 & 102295 & 18323 & 18.43 & $79,360,487$ & \begin{tabular}{|l|}
$14,626,138$ \\
$(11,785,032-$ \\
$17,689,453)$ \\
\end{tabular} & 14.85 & 22.29 & 99.46 & 2768.58 & 0.010 & 0.038 \\
\hline Turkey & 4 & 13332 & 3356 & 22.40 & $78,271,472$ & \begin{tabular}{|l|}
$17,533,182$ \\
$(14,503,704-$ \\
$20,765,422)$
\end{tabular} & 18.53 & 26.53 & 95.65 & 68.90 & 0.001 & 0.009 \\
\hline Israel & 2 & 3008 & 343 & $\mathrm{~N} / \mathrm{C}$ & $\mathrm{N} / \mathrm{C}$ & $\mathrm{N} / \mathrm{C}$ & $\mathrm{N} / \mathrm{C}$ & $\mathrm{N} / \mathrm{C}$ & $\mathrm{N} / \mathrm{C}$ & $\mathrm{N} / \mathrm{C}$ & $\mathrm{N} / \mathrm{C}$ & $\mathrm{N} / \mathrm{C}$ \\
\hline Saudi Arabia & 2 & 3308 & 1161 & $\mathrm{~N} / \mathrm{C}$ & $\mathrm{N} / \mathrm{C}$ & $\mathrm{N} / \mathrm{C}$ & $\mathrm{N} / \mathrm{C}$ & N/C & $\mathrm{N} / \mathrm{C}$ & $\mathrm{N} / \mathrm{C}$ & $\mathrm{N} / \mathrm{C}$ & $\mathrm{N} / \mathrm{C}$ \\
\hline Poland & 1 & 850 & 302 & $\mathrm{~N} / \mathrm{C}$ & $\mathrm{N} / \mathrm{C}$ & $\mathrm{N} / \mathrm{C}$ & $\mathrm{N} / \mathrm{C}$ & N/C & $\mathrm{N} / \mathrm{C}$ & $\mathrm{N} / \mathrm{C}$ & $\mathrm{N} / \mathrm{C}$ & $\mathrm{N} / \mathrm{C}$ \\
\hline Romania & 1 & 184 & 57 & $\mathrm{~N} / \mathrm{C}$ & $\mathrm{N} / \mathrm{C}$ & $\mathrm{N} / \mathrm{C}$ & $\mathrm{N} / \mathrm{C}$ & $\mathrm{N} / \mathrm{C}$ & $\mathrm{N} / \mathrm{C}$ & $\mathrm{N} / \mathrm{C}$ & $\mathrm{N} / \mathrm{C}$ & $\mathrm{N} / \mathrm{C}$ \\
\hline Russia & 2 & 8877 & 1290 & $\mathrm{~N} / \mathrm{C}$ & $\mathrm{N} / \mathrm{C}$ & $\mathrm{N} / \mathrm{C}$ & $\mathrm{N} / \mathrm{C}$ & N/C & $\mathrm{N} / \mathrm{C}$ & $\mathrm{N} / \mathrm{C}$ & $\mathrm{N} / \mathrm{C}$ & $\mathrm{N} / \mathrm{C}$ \\
\hline Albania & 1 & 845 & 101 & $\mathrm{~N} / \mathrm{C}$ & $\mathrm{N} / \mathrm{C}$ & $\mathrm{N} / \mathrm{C}$ & $\mathrm{N} / \mathrm{C}$ & N/C & $\mathrm{N} / \mathrm{C}$ & $\mathrm{N} / \mathrm{C}$ & $\mathrm{N} / \mathrm{C}$ & $\mathrm{N} / \mathrm{C}$ \\
\hline Italy & 2 & 2032 & 304 & $\mathrm{~N} / \mathrm{C}$ & $\mathrm{N} / \mathrm{C}$ & $\mathrm{N} / \mathrm{C}$ & $\mathrm{N} / \mathrm{C}$ & N/C & $\mathrm{N} / \mathrm{C}$ & $\mathrm{N} / \mathrm{C}$ & $\mathrm{N} / \mathrm{C}$ & $\mathrm{N} / \mathrm{C}$ \\
\hline Greece & 1 & 700 & 241 & $\mathrm{~N} / \mathrm{C}$ & $\mathrm{N} / \mathrm{C}$ & $\mathrm{N} / \mathrm{C}$ & $\mathrm{N} / \mathrm{C}$ & N/C & $\mathrm{N} / \mathrm{C}$ & $\mathrm{N} / \mathrm{C}$ & $\mathrm{N} / \mathrm{C}$ & $\mathrm{N} / \mathrm{C}$ \\
\hline Spain & 3 & 5365 & 640 & $\mathrm{~N} / \mathrm{C}$ & $\mathrm{N} / \mathrm{C}$ & $\mathrm{N} / \mathrm{C}$ & $\mathrm{N} / \mathrm{C}$ & N/C & $\mathrm{N} / \mathrm{C}$ & $\mathrm{N} / \mathrm{C}$ & $\mathrm{N} / \mathrm{C}$ & $\mathrm{N} / \mathrm{C}$ \\
\hline Switzerland & 2 & 7736 & 1299 & $\mathrm{~N} / \mathrm{C}$ & $\mathrm{N} / \mathrm{C}$ & $\mathrm{N} / \mathrm{C}$ & $\mathrm{N} / \mathrm{C}$ & N/C & $\mathrm{N} / \mathrm{C}$ & $\mathrm{N} / \mathrm{C}$ & $\mathrm{N} / \mathrm{C}$ & $\mathrm{N} / \mathrm{C}$ \\
\hline Netherlands & 1 & 502 & 25 & $\mathrm{~N} / \mathrm{C}$ & $\mathrm{N} / \mathrm{C}$ & $\mathrm{N} / \mathrm{C}$ & $\mathrm{N} / \mathrm{C}$ & $\mathrm{N} / \mathrm{C}$ & $\mathrm{N} / \mathrm{C}$ & $\mathrm{N} / \mathrm{C}$ & $\mathrm{N} / \mathrm{C}$ & $\mathrm{N} / \mathrm{C}$ \\
\hline France & 2 & 46377 & 3206 & $\mathrm{~N} / \mathrm{C}$ & $\mathrm{N} / \mathrm{C}$ & $\mathrm{N} / \mathrm{C}$ & $\mathrm{N} / \mathrm{C}$ & $\mathrm{N} / \mathrm{C}$ & $\mathrm{N} / \mathrm{C}$ & $\mathrm{N} / \mathrm{C}$ & $\mathrm{N} / \mathrm{C}$ & $\mathrm{N} / \mathrm{C}$ \\
\hline Germany & 1 & 268 & 23 & $\mathrm{~N} / \mathrm{C}$ & $\mathrm{N} / \mathrm{C}$ & $\mathrm{N} / \mathrm{C}$ & $\mathrm{N} / \mathrm{C}$ & N/C & $\mathrm{N} / \mathrm{C}$ & $\mathrm{N} / \mathrm{C}$ & $\mathrm{N} / \mathrm{C}$ & $\mathrm{N} / \mathrm{C}$ \\
\hline Sweden & 4 & 8120 & 1082 & 16.15 & $9,763,565$ & $\begin{array}{l}1,577,196(938,279- \\
2,338,374)\end{array}$ & 9.61 & 23.95 & 98.34 & 181.20 & 0.010 & 0.038 \\
\hline Finland & 1 & 1700 & 175 & $\mathrm{~N} / \mathrm{C}$ & $\mathrm{N} / \mathrm{C}$ & $\mathrm{N} / \mathrm{C}$ & $\mathrm{N} / \mathrm{C}$ & N/C & $\mathrm{N} / \mathrm{C}$ & $\mathrm{N} / \mathrm{C}$ & $\mathrm{N} / \mathrm{C}$ & $\mathrm{N} / \mathrm{C}$ \\
\hline Norway & 1 & 44997 & 7692 & $\mathrm{~N} / \mathrm{C}$ & $\mathrm{N} / \mathrm{C}$ & $\mathrm{N} / \mathrm{C}$ & $\mathrm{N} / \mathrm{C}$ & N/C & $\mathrm{N} / \mathrm{C}$ & $\mathrm{N} / \mathrm{C}$ & $\mathrm{N} / \mathrm{C}$ & $\mathrm{N} / \mathrm{C}$ \\
\hline UK & 4 & 12467 & 1942 & 14.53 & $65,397,080$ & $\begin{array}{l}9,500,537(5,990,373- \\
13,635,291)\end{array}$ & 9.16 & 20.85 & 98.78 & 246.05 & 0.010 & 0.028 \\
\hline
\end{tabular}




\begin{tabular}{|c|c|c|c|c|c|c|c|c|c|c|c|c|}
\hline \multirow{2}{*}{$\begin{array}{l}\text { Geographical } \\
\text { location }\end{array}$} & \multirow{2}{*}{$\begin{array}{l}\text { No. of } \\
\text { studies }\end{array}$} & \multirow{2}{*}{$\begin{array}{l}\text { No. of } \\
\text { participants }\end{array}$} & \multicolumn{2}{|c|}{\begin{tabular}{|l} 
GORD \\
prevalence
\end{tabular}} & \multirow{2}{*}{$\begin{array}{l}\text { Total estimated } \\
\text { population } \\
\text { size }^{\text {b }}\end{array}$} & \multirow{2}{*}{$\begin{array}{l}\text { Total estimated } \\
\text { prevalence of GORD, } \\
\text { n }^{\mathrm{a}}(95 \% \mathrm{CI})\end{array}$} & \multicolumn{2}{|c|}{ 95\% CI } & \multirow[b]{2}{*}{$\mathrm{I}^{2}$} & \multirow{2}{*}{$\begin{array}{l}\text { Cochran's } \\
\text { Q }\end{array}$} & \multirow[b]{2}{*}{$\mathrm{Chi}^{2}, \mathrm{p}$} & \multirow[b]{2}{*}{$\mathrm{Tau}^{2}$} \\
\hline & & & $\mathrm{n}^{\mathrm{a}}$ & $\%$ & & & LCI & HCI & & & & \\
\hline Denmark & 1 & 48027 & 5387 & $\mathrm{~N} / \mathrm{C}$ & N/C & $\mathrm{N} / \mathrm{C}$ & $\mathrm{N} / \mathrm{C}$ & $\mathrm{N} / \mathrm{C}$ & $\mathrm{N} / \mathrm{C}$ & $\mathrm{N} / \mathrm{C}$ & $\mathrm{N} / \mathrm{C}$ & $\mathrm{N} / \mathrm{C}$ \\
\hline USA & 8 & 19489 & 3527 & 21.04 & $319,929,162$ & $\begin{array}{l}67,313,096 \\
(54,132,014- \\
81,517,950)\end{array}$ & 16.92 & 25.48 & 97.79 & 316.06 & 0.010 & 0.022 \\
\hline Canada & 1 & 1036 & 96 & $\mathrm{~N} / \mathrm{C}$ & $\mathrm{N} / \mathrm{C}$ & $\mathrm{N} / \mathrm{C}$ & $\mathrm{N} / \mathrm{C}$ & $\mathrm{N} / \mathrm{C}$ & $\mathrm{N} / \mathrm{C}$ & $\mathrm{N} / \mathrm{C}$ & $\mathrm{N} / \mathrm{C}$ & $\mathrm{N} / \mathrm{C}$ \\
\hline Argentina & 1 & 839 & 100 & $\mathrm{~N} / \mathrm{C}$ & N/C & $\mathrm{N} / \mathrm{C}$ & $\mathrm{N} / \mathrm{C}$ & N/C & $\mathrm{N} / \mathrm{C}$ & $\mathrm{N} / \mathrm{C}$ & $\mathrm{N} / \mathrm{C}$ & $\mathrm{N} / \mathrm{C}$ \\
\hline Brazil & 1 & 3934 & 1231 & $\mathrm{~N} / \mathrm{C}$ & $\mathrm{N} / \mathrm{C}$ & $\mathrm{N} / \mathrm{C}$ & $\mathrm{N} / \mathrm{C}$ & $\mathrm{N} / \mathrm{C}$ & $\mathrm{N} / \mathrm{C}$ & $\mathrm{N} / \mathrm{C}$ & $\mathrm{N} / \mathrm{C}$ & $\mathrm{N} / \mathrm{C}$ \\
\hline Uruguay & 1 & 1141 & 54 & $\mathrm{~N} / \mathrm{C}$ & N/C & N/C & $\mathrm{N} / \mathrm{C}$ & $\mathrm{N} / \mathrm{C}$ & N/C & $\mathrm{N} / \mathrm{C}$ & N/C & $\mathrm{N} / \mathrm{C}$ \\
\hline Colombia & 1 & 6842 & 820 & $\mathrm{~N} / \mathrm{C}$ & $\mathrm{N} / \mathrm{C}$ & $\mathrm{N} / \mathrm{C}$ & $\mathrm{N} / \mathrm{C}$ & $\mathrm{N} / \mathrm{C}$ & $\mathrm{N} / \mathrm{C}$ & $\mathrm{N} / \mathrm{C}$ & $\mathrm{N} / \mathrm{C}$ & $\mathrm{N} / \mathrm{C}$ \\
\hline Australia & 3 & 2982 & 381 & $\mathrm{~N} / \mathrm{C}$ & N/C & N/C & $\mathrm{N} / \mathrm{C}$ & $\mathrm{N} / \mathrm{C}$ & $\mathrm{N} / \mathrm{C}$ & $\mathrm{N} / \mathrm{C}$ & $\mathrm{N} / \mathrm{C}$ & $\mathrm{N} / \mathrm{C}$ \\
\hline New Zealand & 1 & 778 & 122 & $\mathrm{~N} / \mathrm{C}$ & $\mathrm{N} / \mathrm{C}$ & $\mathrm{N} / \mathrm{C}$ & $\mathrm{N} / \mathrm{C}$ & $\mathrm{N} / \mathrm{C}$ & $\mathrm{N} / \mathrm{C}$ & $\mathrm{N} / \mathrm{C}$ & $\mathrm{N} / \mathrm{C}$ & $\mathrm{N} / \mathrm{C}$ \\
\hline Nigeria & 1 & 410 & 108 & $\mathrm{~N} / \mathrm{C}$ & N/C & N/C & N/C & $\mathrm{N} / \mathrm{C}$ & $\mathrm{N} / \mathrm{C}$ & $\mathrm{N} / \mathrm{C}$ & $\mathrm{N} / \mathrm{C}$ & $\mathrm{N} / \mathrm{C}$ \\
\hline Côte d'Ivoire & 1 & 1940 & 178 & $\mathrm{~N} / \mathrm{C}$ & $\mathrm{N} / \mathrm{C}$ & $\mathrm{N} / \mathrm{C}$ & $\mathrm{N} / \mathrm{C}$ & $\mathrm{N} / \mathrm{C}$ & $\mathrm{N} / \mathrm{C}$ & $\mathrm{N} / \mathrm{C}$ & $\mathrm{N} / \mathrm{C}$ & $\mathrm{N} / \mathrm{C}$ \\
\hline
\end{tabular}

Table 1. Pooled prevalence of GORD according to geographical location. GORD, gastro-oesophageal reflux disease; CI, confidence interval; LCI, lower confidence interval; $\mathrm{HCI}$, higher confidence interval; N/C, not computable due to inadequate number of studies. ${ }^{a}$ Number of subjects with GORD. ${ }^{b}$ According to United Nations 2017 Revision of World Population Prospects. ${ }^{c}$ Results from 102 studies reported in 96 records. ${ }^{\mathrm{d} I n a d e q u a t e}$ number of studies to provide estimation of prevalence of GORD in Africa.

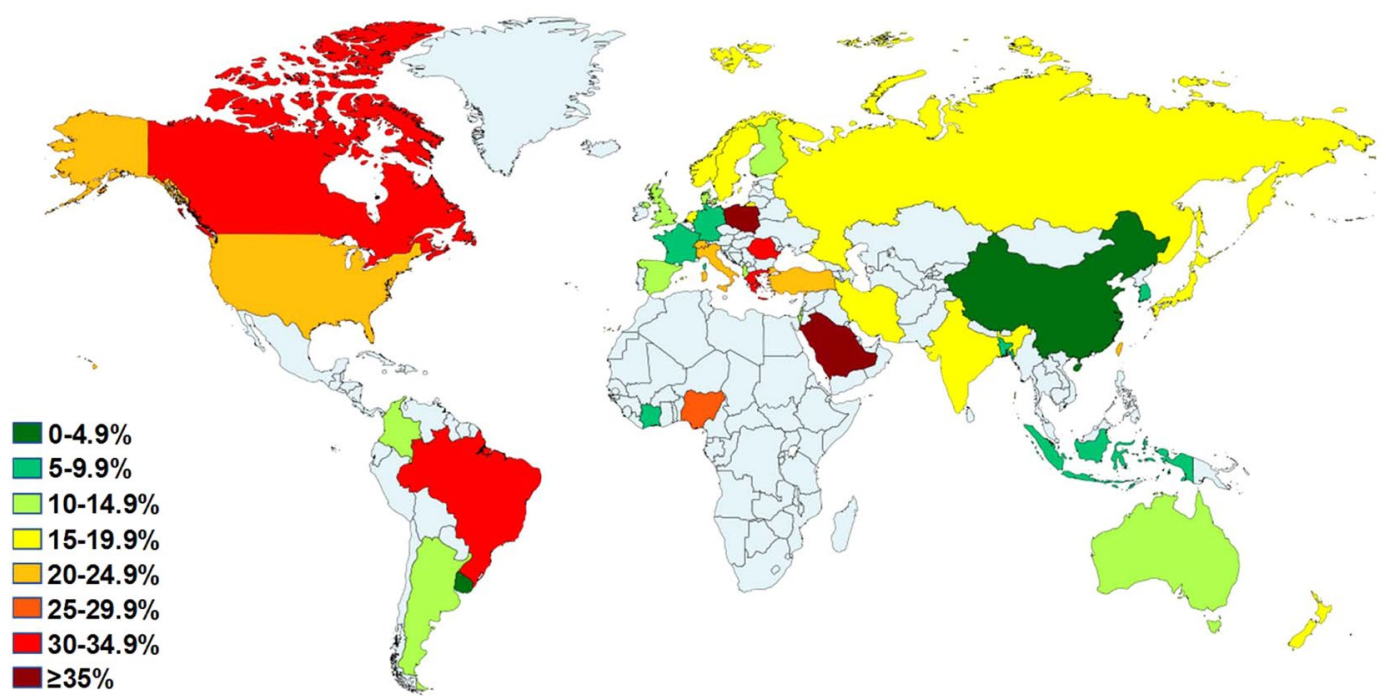

Figure 2. Distribution of GORD prevalence according to country.

The pooled prevalence of GORD according to smoking habits showed that subjects who currently smoke had a higher prevalence of GORD (18.40\% (95\% CI 14.57\%-122.57\%)) compared with ex-smokers (16.83\% (95\% CI $7.49 \%-128.72 \%)$ ) and non-smokers (15.55\% (11.63\%-19.91\%)). However, the OR and RR in current smokers compared with non-smokers was insignificant $(\mathrm{OR}=1.04(95 \% \mathrm{CI} 1.00-1.09 ; \mathrm{P}=0.0652)$ and $\mathrm{RR}=1.04(95 \%$ CI 1.00-1.08; $\mathrm{P}=0.0650)$ ).

Subjects with a low income had a significantly higher prevalence of GORD (11.69\% (95\% CI 4.74\%-120.96\%)) than those with a medium income (8.42\% (95\% CI 1.54\%-119.12\%)) and those with a high income $(7.68 \%(95 \%$ CI $3.86 \%-112.58 \%)$ ). The OR and RR of GORD in those with a low income compared with medium income level was 1.29 (95\% CI 1.33-1.47) and 1.27 (95\% CI 1.12-1.44), respectively. However, the OR and RR in those with a low income compared with a high income was insignificant $(\mathrm{OR}=1.06$ (95\% CI 0.88-1.27); RR $=1.05$ (95\% CI 0.89-1.25)), and subjects with a medium income actually had low OR and RR compared with those with a high income $(\mathrm{OR}=0.82$ (95\% CI 0.67-0.99); $\mathrm{RR}=0.83$ (95\% CI 0.69-0.99)).

The effect of dietary intake of certain food and drinks (spicy food, sweet food, meat/fish, carbonated drinks, fatty food, fried food, and coffee/tea) on the pooled prevalence of GORD was also investigated. However, a limited number of studies have been conducted on the effects of spicy food, sweet food, meat/fish, fatty food and fried food, therefore, meta-analysis was not conducted on these sub-groups (Table 2).

Subjects with a moderate/high intake of carbonated drinks had a higher pooled prevalence of GORD than those with low/none intake (18.60\% (95\% CI 9.55\%-129.68\%) vs $14.54 \%$ (95\% CI 6.49\%-124.91\%), respectively). 


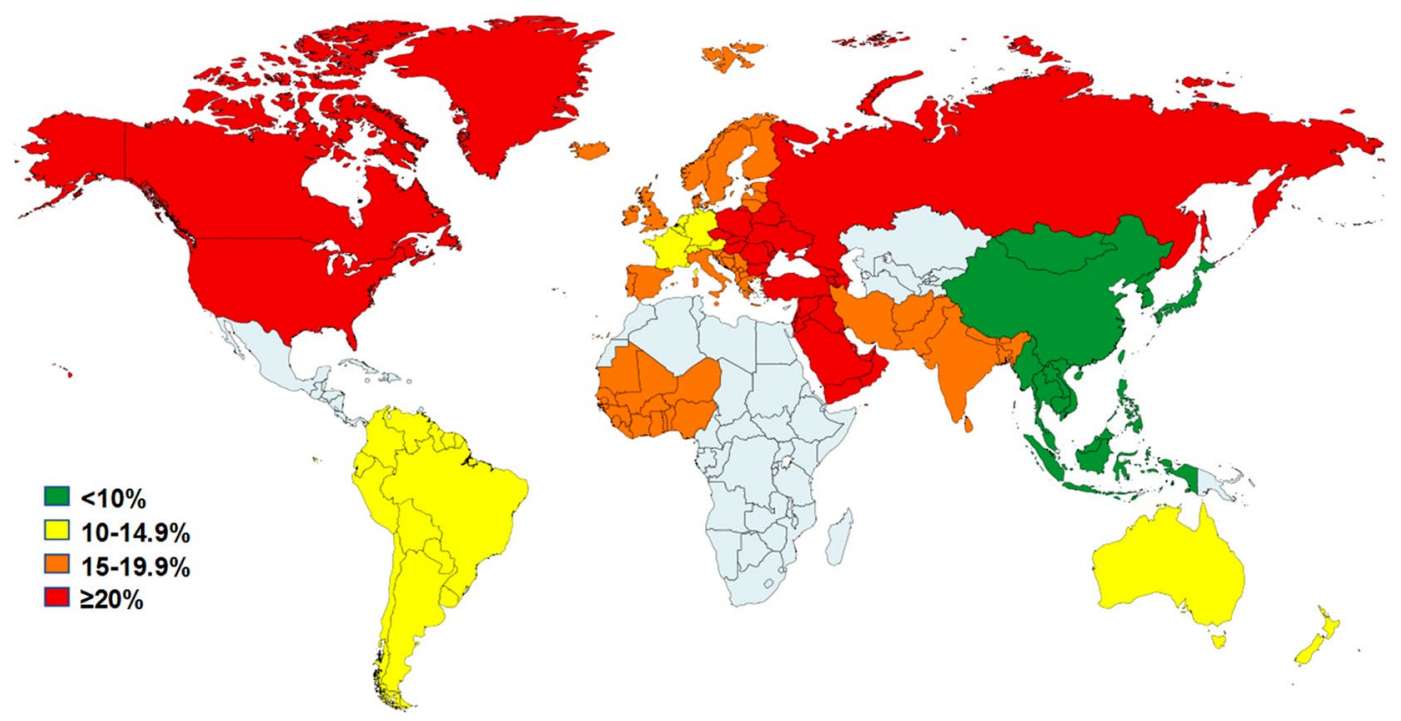

Figure 3. Distribution of GORD prevalence according to sub-regions (Northern America, South America, Northern Europe, Western Europe, Southern Europe, Eastern Europe, Western Africa, Western Asia, Southern Asia, Eastern Asia, South-eastern Asia, Australia \& New Zealand).

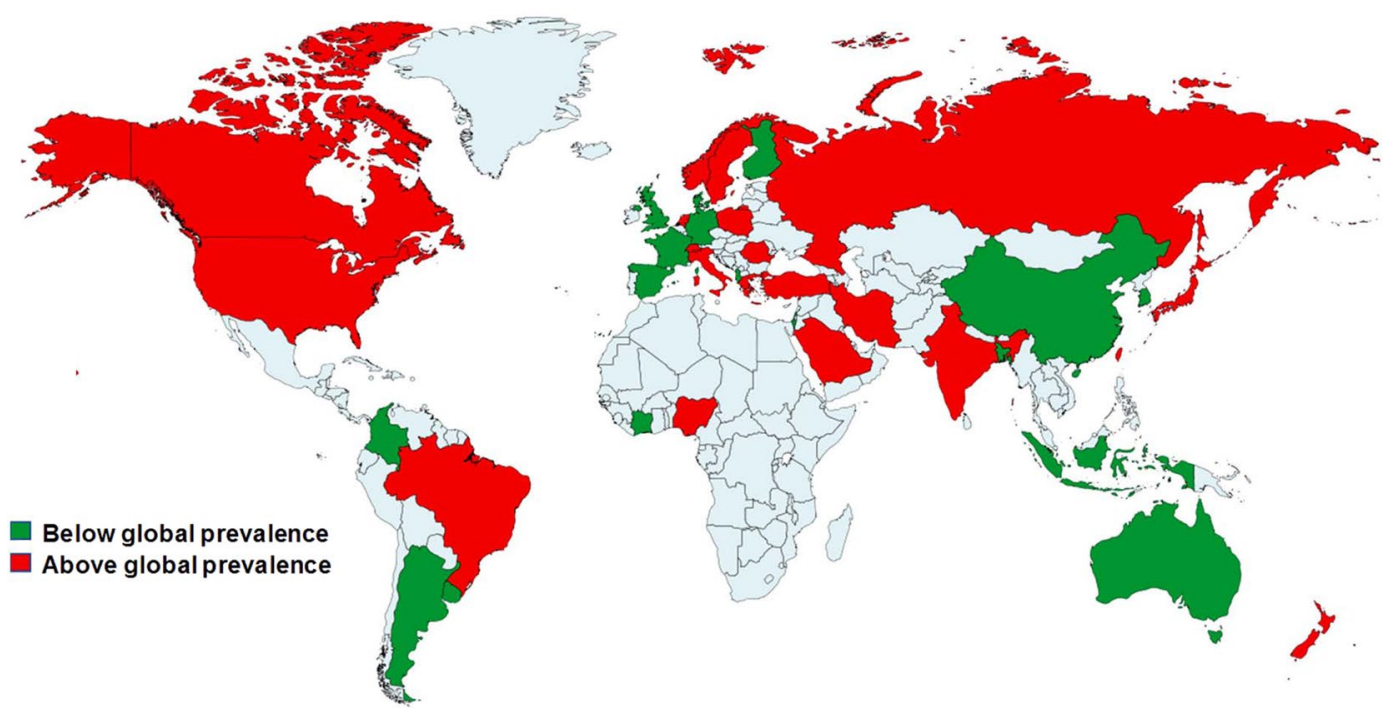

Figure 4. Distribution of GORD prevalence according to global prevalence.

The OR and RR associated with a moderate/high intake of carbonated drinks was 1.29 (95\% CI 1.14-1.46; $\mathrm{P}=0.0001)$ and 1.24 (95\% CI 1.12-1.37; $\mathrm{p}<0.0001)$, respectively.

Pooling prevalence of GORD according to intake of coffee/tea revealed a higher GORD prevalence in subjects with a moderate/high intake of coffee/tea (21.02\% (95\% CI 16.32\%-126.13\%)) than those with a low/none intake (16.92\% (95\% CI 12.69\%-121.61\%)). The OR and RR in those with a moderate/high intake of coffee/tea was 1.47 (95\% CI 1.36-1.59) and 1.38 (95\% CI 1.29-1.47), respectively.

Prevalence of GORD according to study design parameters. Potential sources of heterogeneity were investigated by stratifying pooled prevalence of GORD by study design parameters including criteria used to define GORD, duration of symptoms investigated, instrument used for data collection, and method of data collection. The results of these analyses are shown in Table 3 and the forest plots of pooled prevalence of GORD stratified by study design parameters are presented in Figs. S35-S58. The majority of studies used a definition of at least weekly heartburn and/or acid regurgitation to define GORD (66 studies). From these studies, the pooled prevalence of GORD was $13.45 \%$ (95\% CI 11.79\%-115.20). The lowest prevalence of GORD was achieved when the Montreal definition was used (12.07\% (95\% CI 7.53\%-117.46\%)), and a definition of GERDQ score $\geq 8$ produced the highest prevalence (17.03\% (95\% CI 9.24\%-126.52\%)). 


\begin{tabular}{|c|c|c|c|c|c|c|c|c|c|c|}
\hline \multirow[b]{2}{*}{ Risk factor } & \multirow{2}{*}{$\begin{array}{l}\text { No. of } \\
\text { studies }\end{array}$} & \multirow{2}{*}{$\begin{array}{l}\text { No. of } \\
\text { participants }\end{array}$} & \multicolumn{2}{|c|}{ GORD prevalence } & \multicolumn{2}{|c|}{$95 \% \mathrm{CI}$} & \multirow[b]{2}{*}{$I^{2}$} & \multirow{2}{*}{$\begin{array}{l}\text { Cochran's } \\
\text { Q }\end{array}$} & \multirow[b]{2}{*}{$\mathrm{Chi}^{2}, \mathbf{p}$} & \multirow[b]{2}{*}{$\mathrm{Tau}^{2}$} \\
\hline & & & $\mathbf{n}^{\mathrm{a}}$ & $\%$ & LCI & HCI & & & & \\
\hline \multicolumn{11}{|l|}{ Age group (years) } \\
\hline $18-34$ & 15 & 15065 & 1733 & 8.70 & 4.39 & 14.23 & 99.04 & 1460.27 & 0.010 & 0.112 \\
\hline $35-59$ & 21 & 55851 & 7362 & 14.53 & 11.09 & 18.33 & 99.24 & 2638.42 & 0.010 & 0.056 \\
\hline$\geq 60$ & 17 & 17663 & 2383 & 13.12 & 9.59 & 17.11 & 98.10 & 840.21 & 0.010 & 0.052 \\
\hline \multicolumn{11}{|l|}{ Alcohol intake } \\
\hline None/Low & 21 & 41655 & 4998 & 15.95 & 11.01 & 21.60 & 99.50 & 4028.78 & 0.010 & 0.111 \\
\hline Moderate/High & 21 & 24445 & 3117 & 15.56 & 11.63 & 19.93 & 98.64 & 1470.65 & 0.010 & 0.066 \\
\hline \multicolumn{11}{|l|}{ BMI } \\
\hline$<18.5$ & 6 & 2506 & 110 & 6.64 & 3.40 & 10.82 & 88.55 & 43.66 & 0.001 & 0.026 \\
\hline $18.5-29.9$ & 14 & 10244 & 1830 & 17.20 & 11.80 & 23.37 & 98.25 & 628.66 & 0.010 & 0.070 \\
\hline$\geq 30.0$ & 12 & 3423 & 934 & 22.63 & 17.33 & 28.41 & 92.95 & 156.10 & 0.010 & 0.048 \\
\hline \multicolumn{11}{|l|}{ Education level } \\
\hline Low & 21 & 24609 & 3582 & 16.78 & 12.32 & 21.77 & 98.83 & 1620.47 & 0.010 & 0.079 \\
\hline Medium & 15 & 23428 & 1747 & 11.52 & 7.33 & 16.48 & 98.98 & 1375.18 & 0.010 & 0.077 \\
\hline High & 18 & 16159 & 1433 & 8.98 & 5.56 & 13.09 & 98.46 & 1037.44 & 0.010 & 0.072 \\
\hline \multicolumn{11}{|l|}{ Income level } \\
\hline Low & 6 & 11034 & 671 & 11.69 & 4.74 & 20.96 & 98.60 & 357.66 & 0.010 & 0.091 \\
\hline Medium & 4 & 7704 & 368 & 8.42 & 1.54 & 19.12 & 98.92 & 278.77 & 0.010 & 0.093 \\
\hline High & 6 & 2615 & 151 & 7.68 & 3.86 & 12.58 & 92.78 & 69.21 & 0.001 & 0.036 \\
\hline \multicolumn{11}{|l|}{ Marriage status } \\
\hline Single & 12 & 11657 & 1150 & 12.85 & 6.59 & 20.71 & 98.98 & 1075.90 & 0.010 & 0.128 \\
\hline Married & 12 & 28768 & 4166 & 15.98 & 10.48 & 22.35 & 99.40 & 1836.17 & 0.010 & 0.080 \\
\hline Divorced/separated/widowed & 6 & 1538 & 423 & 22.95 & 13.19 & 34.38 & 95.35 & 107.57 & 0.010 & 0.090 \\
\hline NSAIDs/aspirin use & & & & & & & & & & \\
\hline Users & 10 & 3574 & 741 & 24.47 & 18.17 & 31.35 & 94.38 & 160.09 & 0.010 & 0.054 \\
\hline Non-users & 10 & 14419 & 2192 & 17.34 & 13.36 & 21.72 & 97.15 & 316.08 & 0.010 & 0.030 \\
\hline Area of domicile & & & & & & & & & & \\
\hline Rural & 8 & 12387 & 870 & 11.70 & 6.26 & 18.46 & 98.59 & 495.59 & 0.010 & 0.070 \\
\hline Suburban & 2 & 1376 & 48 & $\mathrm{~N} / \mathrm{C}$ & $\mathrm{N} / \mathrm{C}$ & $\mathrm{N} / \mathrm{C}$ & $\mathrm{N} / \mathrm{C}$ & $\mathrm{N} / \mathrm{C}$ & $\mathrm{N} / \mathrm{C}$ & $\mathrm{N} / \mathrm{C}$ \\
\hline Urban & 8 & 11528 & 1660 & 13.43 & 6.68 & 21.95 & 99.28 & 969.49 & 0.010 & 0.100 \\
\hline Smoking habits & & & & & & & & & & \\
\hline Current smokers & 28 & 28574 & 3738 & 18.40 & 14.57 & 22.57 & 98.54 & 1844.55 & 0.010 & 0.072 \\
\hline Ex-smokers & 5 & 990 & 144 & 16.83 & 7.49 & 28.72 & 94.26 & 69.63 & 0.001 & 0.093 \\
\hline Non-smokers & 28 & 54787 & 6921 & 15.55 & 11.63 & 19.91 & 99.43 & 4699.24 & 0.010 & 0.093 \\
\hline Spicy food & & & & & & & & & & \\
\hline Low/none & 3 & 3397 & 274 & $\mathrm{~N} / \mathrm{C}$ & $\mathrm{N} / \mathrm{C}$ & $\mathrm{N} / \mathrm{C}$ & $\mathrm{N} / \mathrm{C}$ & $\mathrm{N} / \mathrm{C}$ & $\mathrm{N} / \mathrm{C}$ & $\mathrm{N} / \mathrm{C}$ \\
\hline Moderate/high & 3 & 2813 & 280 & $\mathrm{~N} / \mathrm{C}$ & $\mathrm{N} / \mathrm{C}$ & $\mathrm{N} / \mathrm{C}$ & $\mathrm{N} / \mathrm{C}$ & $\mathrm{N} / \mathrm{C}$ & $\mathrm{N} / \mathrm{C}$ & $\mathrm{N} / \mathrm{C}$ \\
\hline Sweet food & & & & & & & & & & \\
\hline Low/none & 2 & 459 & 136 & $\mathrm{~N} / \mathrm{C}$ & $\mathrm{N} / \mathrm{C}$ & $\mathrm{N} / \mathrm{C}$ & $\mathrm{N} / \mathrm{C}$ & $\mathrm{N} / \mathrm{C}$ & $\mathrm{N} / \mathrm{C}$ & $\mathrm{N} / \mathrm{C}$ \\
\hline Moderate/high & 2 & 2177 & 613 & $\mathrm{~N} / \mathrm{C}$ & $\mathrm{N} / \mathrm{C}$ & $\mathrm{N} / \mathrm{C}$ & $\mathrm{N} / \mathrm{C}$ & $\mathrm{N} / \mathrm{C}$ & $\mathrm{N} / \mathrm{C}$ & $\mathrm{N} / \mathrm{C}$ \\
\hline Meat/fish & & & & & & & & & & \\
\hline Low/none & 3 & 3443 & 313 & $\mathrm{~N} / \mathrm{C}$ & $\mathrm{N} / \mathrm{C}$ & $\mathrm{N} / \mathrm{C}$ & $\mathrm{N} / \mathrm{C}$ & $\mathrm{N} / \mathrm{C}$ & $\mathrm{N} / \mathrm{C}$ & $\mathrm{N} / \mathrm{C}$ \\
\hline Moderate/high & 3 & 2234 & 568 & $\mathrm{~N} / \mathrm{C}$ & $\mathrm{N} / \mathrm{C}$ & $\mathrm{N} / \mathrm{C}$ & $\mathrm{N} / \mathrm{C}$ & $\mathrm{N} / \mathrm{C}$ & $\mathrm{N} / \mathrm{C}$ & $\mathrm{N} / \mathrm{C}$ \\
\hline Carbonated drinks & & & & & & & & & & \\
\hline Low/none & 5 & 6837 & 944 & 14.54 & 6.49 & 24.91 & 99.01 & 403.33 & 0.010 & 0.082 \\
\hline Moderate/high & 5 & 2644 & 452 & 18.60 & 9.55 & 29.68 & 97.51 & 160.71 & 0.010 & 0.081 \\
\hline Fatty food & & & & & & & & & & \\
\hline Low/none & 2 & 1355 & 118 & $\mathrm{~N} / \mathrm{C}$ & $\mathrm{N} / \mathrm{C}$ & $\mathrm{N} / \mathrm{C}$ & $\mathrm{N} / \mathrm{C}$ & $\mathrm{N} / \mathrm{C}$ & $\mathrm{N} / \mathrm{C}$ & $\mathrm{N} / \mathrm{C}$ \\
\hline Moderate/high & 2 & 395 & 42 & $\mathrm{~N} / \mathrm{C}$ & $\mathrm{N} / \mathrm{C}$ & $\mathrm{N} / \mathrm{C}$ & $\mathrm{N} / \mathrm{C}$ & $\mathrm{N} / \mathrm{C}$ & $\mathrm{N} / \mathrm{C}$ & $\mathrm{N} / \mathrm{C}$ \\
\hline Fried food & & & & & & & & & & \\
\hline Low/none & 3 & 2799 & 204 & $\mathrm{~N} / \mathrm{C}$ & $\mathrm{N} / \mathrm{C}$ & $\mathrm{N} / \mathrm{C}$ & $\mathrm{N} / \mathrm{C}$ & $\mathrm{N} / \mathrm{C}$ & $\mathrm{N} / \mathrm{C}$ & $\mathrm{N} / \mathrm{C}$ \\
\hline Moderate/high & 3 & 1984 & 378 & $\mathrm{~N} / \mathrm{C}$ & $\mathrm{N} / \mathrm{C}$ & $\mathrm{N} / \mathrm{C}$ & $\mathrm{N} / \mathrm{C}$ & $\mathrm{N} / \mathrm{C}$ & $\mathrm{N} / \mathrm{C}$ & $\mathrm{N} / \mathrm{C}$ \\
\hline Coffee/tea & & & & & & & & & & \\
\hline Low/none & 14 & 7104 & 1018 & 16.92 & 12.69 & 21.61 & 95.62 & 296.68 & 0.010 & 0.045 \\
\hline Moderate/high & 14 & 17174 & 3387 & 21.02 & 16.32 & 26.13 & 98.28 & 756.60 & 0.010 & 0.050 \\
\hline
\end{tabular}

Table 2. Pooled prevalence of GORD according to risk factors. BMI, body mass index; NSAID, nonsteroidal antiinflammatory drug; N/C, not computable due to inadequate number of studies. ${ }^{a}$ Number of subjects with GORD. 
The majority of studies assessed GORD symptoms during the previous 12 months (38 studies). The highest prevalence of GORD was also seen in studies which assessed GORD symptoms during the previous 12 months (15.23\% (95\% CI 13.25\%-117.32\%)), and the lowest prevalence was observed in studies which assessed GORD symptoms during the previous 1 month (5.20\% (95\% CI 1.51\%-110.64\%)).

The pooled prevalence of GORD according to the instrument used for data collection ranged from 5.17\% (95\% CI 2.46\%-18.76\%) in studies using the Reflux Disease Questionnaire (RDQ), to $26.90 \%$ (95\% CI 25.24\%$128.69 \%$ ) in studies using the Quality of life and Utility Evaluation Survey Technology (QUEST).

The most frequently used method of data collection were face-to-face interviews (31 studies) which gave a pooled GORD prevalence of $16.98 \%$ (95\% CI 13.74\%-120.49\%). This was also the highest prevalence of GORD when stratified by method of data collection. The lowest GORD prevalence was seen when telephone interviews were used to collect data (9.57\% (95\% CI 7.80\%-111.49\%)).

\section{Discussion}

This comprehensive systematic review has demonstrated the significant global burden of GORD with approximately 1.03 billion individuals suffering from the condition globally. It has also confirmed substantial variations in the pooled prevalence of GORD between regions and countries. The region with the highest pooled prevalence of GORD was North America (19.55\%) and the lowest pooled prevalence was in Latin America and the Caribbean (South America) (12.88\%) (Fig. 3). This is in contrast with the systematic review conducted by El-Serag et al. 2013, which found the prevalence of GORD in South America to be significantly greater at $23.0 \%{ }^{20}$. However, their systematic review was limited to a single study from the region. One explanation for this distribution may be the high rate of obesity in North America ${ }^{123}$. This systematic review has identified that obese individuals are significantly more at risk of suffering from GORD and females are also more at risk than males. The prevalence of GORD was also higher in those who had a high intake of food and drinks associated with obesity, such as fatty food and carbonated drinks, and was significantly higher in those with a moderate to high intake of coffee/tea. These factors may also explain the high prevalence of GORD in North America and Europe where intake of these foods is high (Fig. 3). Additionally, individuals living in an urban area have been identified as having a higher prevalence of GORD compared with those living in a rural area. Therefore, high levels of urbanisation may contribute to increased prevalence of GORD in Western regions, such as North America and Europe, compared to regions where rural areas predominate, such as in Asia and Latin America and the Caribbean. Moreover, individuals had significantly higher odds of suffering from GORD than married or single individuals, which may further explain the high prevalence of GORD in Europe and North America where divorce rates are high ${ }^{124}$.

Other factors which were associated with an increased risk of GORD were education level, age, and intake of NSAIDs/aspirin. The odds of GORD were significantly higher in those with a low level of education compared with those with a medium or high level of education. Similarly, the prevalence of GORD in individuals with a lower income was significantly greater than those with a medium income. These trends demonstrate the relationship between socioeconomic status and risk of GORD. A potential explanation for this trend may be an increased awareness of health issues in educated individuals compared with those with low or no education. Additionally, individuals with a higher income level may be able to afford a wider range of treatment options, as well as healthier food options, which may prevent or alleviate GORD symptoms. However, those with a medium level of education were not more at risk of suffering from GORD than those with a higher education level. Individuals with a medium income level were also not more at risk than those with a high-income level. Furthermore, the prevalence of GORD was higher in those aged 35-59 years than those aged 18-34 years but was slightly lower in those aged $\geq 60$ years compared with those aged $35-59$ years. GORD prevalence was also significantly higher in those who use NSAIDs/aspirin. Intake of these classes of drugs have been associated with an increase in gastric acid secretion, reduction in lower oesophageal sphincter pressure, and a delay in gastric emptying, leading to an increase in the risk of GORD ${ }^{125-127}$. Moreover, those with a moderate/high intake of spicy food had a significantly higher prevalence of GORD than those with low/no intake, potentially due to a slowed rate of digestion and irritation of the oesophagus caused by intake of spices ${ }^{128,129}$.

Interestingly, although current smokers had a higher prevalence of GORD than non-smokers and ex-smokers, the OR and RR was not significant, and was actually higher in ex-smokers compared with current smokers and non-smokers.

As one of the most comprehensive and up-to-date systematic reviews on the global prevalence and risk factors of GORD, this review has several strengths. We pooled data according to region, sub-region, and country to analyse the distribution of GORD around the world. We also identified two studies from Africa; a region from which studies have not been included in previous systematic reviews. Furthermore, we have comprehensively investigated how risk factors affect the prevalence of GORD, including more risk factors than previous systematic reviews. Articles published in a language other than English $(n=5)$ were translated and included in this review.

This review also has some limitations. Significant heterogeneity was demonstrated between studies in most analyses. Potential sources of heterogeneity were investigated by stratifying the pooled prevalence of GORD by study design parameters; however, significant heterogeneity was still present between studies. Substantial variations in GORD prevalence also existed within the parameters analysed. From the 102 studies included in this review, a total of 14 different definitions were used to define GORD, as well as 13 different instruments used to collect data from subjects. Although many of the instruments assessed similar symptoms, frequency of symptoms, and severity of symptoms, differences in the design may affect how subjects interpret the components of instruments. Additionally, many authors were required to translate the instruments to the language spoken in the location of interest, potentially leading to translation issues and further interpretation issues. Also, different cut-off scores were used to diagnose GORD for the same instrument e.g. for the GERDQ instrument, cut-off scores of $\geq 7, \geq 8$, and $\geq 12$ were used by different studies, leading to a wide range in GORD prevalence for the 


\begin{tabular}{|c|c|c|c|c|c|c|c|c|c|c|}
\hline \multirow[b]{2}{*}{ Study design parameter } & \multirow{2}{*}{$\begin{array}{l}\text { No. of } \\
\text { studies }\end{array}$} & \multirow{2}{*}{$\begin{array}{l}\text { No. of } \\
\text { participants }\end{array}$} & \multicolumn{2}{|c|}{ GORD prevalence } & \multicolumn{2}{|c|}{ 95\% CI } & \multirow[b]{2}{*}{$\mathbf{I}^{2}$} & \multirow[b]{2}{*}{ Cochran's Q } & \multirow[b]{2}{*}{$\mathrm{Chi}^{2}, \mathbf{p}$} & \multirow[b]{2}{*}{$\mathrm{Tau}^{2}$} \\
\hline & & & $\mathbf{n}^{\mathrm{a}}$ & $\%$ & LCI & HCI & & & & \\
\hline \multicolumn{11}{|l|}{ Criteria used to define GORD } \\
\hline At least weekly heartburn and/or acid regurgitation & 66 & 275040 & 34745 & 13.45 & 11.79 & 15.20 & 99.40 & 11783.76 & 0.010 & 0.046 \\
\hline Montreal definition & 10 & 124993 & 16835 & 12.07 & 7.53 & 17.46 & 99.82 & 4912.40 & 0.010 & 0.058 \\
\hline GERDQ score $\geq 7$ & 1 & 278 & 26 & $\mathrm{~N} / \mathrm{C}$ & $\mathrm{N} / \mathrm{C}$ & $\mathrm{N} / \mathrm{C}$ & $\mathrm{N} / \mathrm{C}$ & $\mathrm{N} / \mathrm{C}$ & $\mathrm{N} / \mathrm{C}$ & $\mathrm{N} / \mathrm{C}$ \\
\hline GERDQ score $\geq 8$ & 8 & 24929 & 4786 & 17.03 & 9.24 & 26.52 & 99.67 & 2096.59 & 0.010 & 0.106 \\
\hline GERDQ score $\geq 12$ & 1 & 1238 & 310 & $\mathrm{~N} / \mathrm{C}$ & $\mathrm{N} / \mathrm{C}$ & $\mathrm{N} / \mathrm{C}$ & $\mathrm{N} / \mathrm{C}$ & $\mathrm{N} / \mathrm{C}$ & $\mathrm{N} / \mathrm{C}$ & $\mathrm{N} / \mathrm{C}$ \\
\hline Diagnosed by endoscopy & 5 & 21061 & 2445 & 12.87 & 4.78 & 23.79 & 99.75 & 1606.16 & 0.010 & 0.099 \\
\hline Previously diagnosed by a physician & 1 & 8143 & 2238 & $\mathrm{~N} / \mathrm{C}$ & $\mathrm{N} / \mathrm{C}$ & $\mathrm{N} / \mathrm{C}$ & $\mathrm{N} / \mathrm{C}$ & $\mathrm{N} / \mathrm{C}$ & $\mathrm{N} / \mathrm{C}$ & $\mathrm{N} / \mathrm{C}$ \\
\hline Currently undergoing treatment for GORD & 3 & 13626 & 2882 & $\mathrm{~N} / \mathrm{C}$ & $\mathrm{N} / \mathrm{C}$ & $\mathrm{N} / \mathrm{C}$ & $\mathrm{N} / \mathrm{C}$ & $\mathrm{N} / \mathrm{C}$ & $\mathrm{N} / \mathrm{C}$ & $\mathrm{N} / \mathrm{C}$ \\
\hline FSSG score $\geq 8$ & 1 & 9643 & 2210 & $\mathrm{~N} / \mathrm{C}$ & $\mathrm{N} / \mathrm{C}$ & $\mathrm{N} / \mathrm{C}$ & $\mathrm{N} / \mathrm{C}$ & $\mathrm{N} / \mathrm{C}$ & $\mathrm{N} / \mathrm{C}$ & $\mathrm{N} / \mathrm{C}$ \\
\hline FSSG score $>10$ & 1 & 1130 & 316 & $\mathrm{~N} / \mathrm{C}$ & $\mathrm{N} / \mathrm{C}$ & $\mathrm{N} / \mathrm{C}$ & $\mathrm{N} / \mathrm{C}$ & $\mathrm{N} / \mathrm{C}$ & $\mathrm{N} / \mathrm{C}$ & $\mathrm{N} / \mathrm{C}$ \\
\hline QUEST score $\geq 6$ & 2 & 6628 & 1271 & $\mathrm{~N} / \mathrm{C}$ & $\mathrm{N} / \mathrm{C}$ & $\mathrm{N} / \mathrm{C}$ & $\mathrm{N} / \mathrm{C}$ & $\mathrm{N} / \mathrm{C}$ & $\mathrm{N} / \mathrm{C}$ & $\mathrm{N} / \mathrm{C}$ \\
\hline RDQ score $\geq 12$ & 1 & 3338 & 83 & $\mathrm{~N} / \mathrm{C}$ & $\mathrm{N} / \mathrm{C}$ & $\mathrm{N} / \mathrm{C}$ & $\mathrm{N} / \mathrm{C}$ & $\mathrm{N} / \mathrm{C}$ & $\mathrm{N} / \mathrm{C}$ & $\mathrm{N} / \mathrm{C}$ \\
\hline GORD-SMQ score $>9$ & 1 & 2603 & 701 & $\mathrm{~N} / \mathrm{C}$ & $\mathrm{N} / \mathrm{C}$ & $\mathrm{N} / \mathrm{C}$ & $\mathrm{N} / \mathrm{C}$ & $\mathrm{N} / \mathrm{C}$ & $\mathrm{N} / \mathrm{C}$ & $\mathrm{N} / \mathrm{C}$ \\
\hline QUEST score $\geq 4$ & 1 & 410 & 108 & $\mathrm{~N} / \mathrm{C}$ & $\mathrm{N} / \mathrm{C}$ & $\mathrm{N} / \mathrm{C}$ & $\mathrm{N} / \mathrm{C}$ & $\mathrm{N} / \mathrm{C}$ & $\mathrm{N} / \mathrm{C}$ & $\mathrm{N} / \mathrm{C}$ \\
\hline \multicolumn{11}{|l|}{ Duration of symptoms } \\
\hline 1 week & 9 & 17558 & 3228 & 12.96 & 6.06 & 21.82 & 99.50 & 1404.15 & 0.010 & 0.109 \\
\hline 1 month & 4 & 22601 & 962 & 5.20 & 1.51 & 10.64 & 99.29 & 422.66 & 0.010 & 0.040 \\
\hline 3 months & 9 & 54860 & 6165 & 10.28 & 7.89 & 12.94 & 98.83 & 1192.54 & 0.010 & 0.026 \\
\hline 6 months & 2 & 20741 & 2152 & $\mathrm{~N} / \mathrm{C}$ & $\mathrm{N} / \mathrm{C}$ & $\mathrm{N} / \mathrm{C}$ & $\mathrm{N} / \mathrm{C}$ & $\mathrm{N} / \mathrm{C}$ & $\mathrm{N} / \mathrm{C}$ & $\mathrm{N} / \mathrm{C}$ \\
\hline 12 months & 38 & 171051 & 29199 & 15.23 & 13.25 & 17.32 & 99.17 & 4441.08 & 0.010 & 0.031 \\
\hline \multicolumn{11}{|l|}{ Instrument used for data collection } \\
\hline GERDQ & 10 & 26445 & 5122 & 16.97 & 10.14 & 25.09 & 99.58 & 2150.78 & 0.010 & 0.100 \\
\hline Mayo Reflux Questionnaire & 18 & 78401 & 8643 & 16.96 & 12.95 & 21.39 & 99.54 & 3736.01 & 0.010 & 0.058 \\
\hline FSSG & 2 & 10773 & 2526 & $\mathrm{~N} / \mathrm{C}$ & $\mathrm{N} / \mathrm{C}$ & $\mathrm{N} / \mathrm{C}$ & $\mathrm{N} / \mathrm{C}$ & $\mathrm{N} / \mathrm{C}$ & $\mathrm{N} / \mathrm{C}$ & $\mathrm{N} / \mathrm{C}$ \\
\hline DIGEST questionnaire & 1 & 5581 & 443 & $\mathrm{~N} / \mathrm{C}$ & $\mathrm{N} / \mathrm{C}$ & $\mathrm{N} / \mathrm{C}$ & $\mathrm{N} / \mathrm{C}$ & $\mathrm{N} / \mathrm{C}$ & $\mathrm{N} / \mathrm{C}$ & $\mathrm{N} / \mathrm{C}$ \\
\hline QUEST & 4 & 8038 & 1638 & 22.52 & 17.27 & 28.25 & 98.23 & 56.39 & 0.001 & 0.017 \\
\hline RDQ & 4 & 6267 & 279 & 5.17 & 2.46 & 8.76 & 96.30 & 81.05 & 0.010 & 0.019 \\
\hline GHQ 28 & 1 & 1000 & 123 & $\mathrm{~N} / \mathrm{C}$ & $\mathrm{N} / \mathrm{C}$ & $\mathrm{N} / \mathrm{C}$ & $\mathrm{N} / \mathrm{C}$ & $\mathrm{N} / \mathrm{C}$ & $\mathrm{N} / \mathrm{C}$ & $\mathrm{N} / \mathrm{C}$ \\
\hline BDQ & 5 & 7718 & 747 & 8.26 & 3.03 & 15.52 & 98.94 & 378.63 & 0.010 & 0.062 \\
\hline GSRS & 2 & 2095 & 457 & $\mathrm{~N} / \mathrm{C}$ & $\mathrm{N} / \mathrm{C}$ & $\mathrm{N} / \mathrm{C}$ & $\mathrm{N} / \mathrm{C}$ & $\mathrm{N} / \mathrm{C}$ & $\mathrm{N} / \mathrm{C}$ & $\mathrm{N} / \mathrm{C}$ \\
\hline GERD-SMQ & 1 & 2603 & 701 & $\mathrm{~N} / \mathrm{C}$ & $\mathrm{N} / \mathrm{C}$ & $\mathrm{N} / \mathrm{C}$ & $\mathrm{N} / \mathrm{C}$ & $\mathrm{N} / \mathrm{C}$ & $\mathrm{N} / \mathrm{C}$ & $\mathrm{N} / \mathrm{C}$ \\
\hline CPQ & 1 & 672 & 78 & $\mathrm{~N} / \mathrm{C}$ & $\mathrm{N} / \mathrm{C}$ & $\mathrm{N} / \mathrm{C}$ & $\mathrm{N} / \mathrm{C}$ & $\mathrm{N} / \mathrm{C}$ & $\mathrm{N} / \mathrm{C}$ & $\mathrm{N} / \mathrm{C}$ \\
\hline SSA-P & 1 & 1395 & 216 & $\mathrm{~N} / \mathrm{C}$ & $\mathrm{N} / \mathrm{C}$ & $\mathrm{N} / \mathrm{C}$ & $\mathrm{N} / \mathrm{C}$ & $\mathrm{N} / \mathrm{C}$ & $\mathrm{N} / \mathrm{C}$ & $\mathrm{N} / \mathrm{C}$ \\
\hline Montreal instrument & 1 & 845 & 101 & $\mathrm{~N} / \mathrm{C}$ & $\mathrm{N} / \mathrm{C}$ & $\mathrm{N} / \mathrm{C}$ & $\mathrm{N} / \mathrm{C}$ & $\mathrm{N} / \mathrm{C}$ & $\mathrm{N} / \mathrm{C}$ & $\mathrm{N} / \mathrm{C}$ \\
\hline \multicolumn{11}{|l|}{ Method of data collection } \\
\hline Self-completed questionnaire & 22 & 116062 & 13478 & 13.12 & 9.97 & 16.63 & 99.58 & 5015.29 & 0.010 & 0.054 \\
\hline Face-to-face interview & 31 & 204028 & 30148 & 16.98 & 13.74 & 20.49 & 99.74 & 13672.67 & 0.010 & 0.077 \\
\hline Telephone interview & 17 & 101892 & 11612 & 9.57 & 7.80 & 11.49 & 98.77 & 1300.40 & 0.010 & 0.017 \\
\hline Postal questionnaire & 20 & 82039 & 12901 & 15.04 & 12.85 & 17.37 & 98.33 & 1136.83 & 0.010 & 0.020 \\
\hline Interview-administered questionnaire & 9 & 11376 & 1369 & 13.49 & 8.96 & 18.76 & 98.26 & 460.11 & 0.010 & 0.046 \\
\hline Endoscopy & 5 & 21061 & 2445 & 12.87 & 4.78 & 23.79 & 99.75 & \begin{tabular}{|l|}
1606.16 \\
\end{tabular} & \begin{tabular}{|l|}
0.010 \\
\end{tabular} & \begin{tabular}{|l}
0.099 \\
\end{tabular} \\
\hline
\end{tabular}

Table 3. Pooled prevalence of GORD according to study design. N/C, not computable due to inadequate number of studies; GerdQ, gastroesophageal reflux disease questionnaire; RDQ, Reflux Disease Questionnaire; BDQ, Bowel Disease Questionnaire; FSSG, Frequency Scale for the Symptoms of GORD; GHQ-28, General Health Questionnaire-28; GSRS, Gastrointestinal Symptom Rating Scale; DIGEST, Domestic/International Gastroenterology Surveillance Study; SSA-P, Subjective Symptom Assessment Profile; GERD-SMQ, GORD Symptom and Medication Questionnaire; CPQ, Chest Pain Questionnaire; QUEST, Quality of life and Utility Evaluation Survey Technology. ${ }^{a}$ Number of subjects with GORD.

same instrument. These factors highlight the need for studies to adhere to a consistent and official definition of GORD to allow for more reliable and comparable estimates of GORD prevalence. A potential candidate for this is the Montreal definition (mild symptoms occurring on $\geq 2 \mathrm{~d}$ of the week or moderate to severe symptoms occurring on $\geq 1 \mathrm{~d}$ of the week) which was approved by a consensus panel of 44 experts in 2006, yet over a decade later, this review identified only 10 studies using this definition ${ }^{130}$. Similarly, to diagnose GORD by the set definition, a standard instrument may be adopted by investigators. 
In conclusion, this systematic review is the most comprehensive review conducted on the prevalence and risk factors of GORD to date, and the first to include studies from all regions of the UN geoscheme. Significant variations in GORD prevalence were found between regions and countries, and we have demonstrated that lifestyle, socioeconomic, and sociodemographic factors may contribute to these variations. These results may have long-reaching implications in clinical practice, research, and industry. The findings of this review will assist clinicians in recognising GORD symptoms in those most at risk, as well as identifying changes in lifestyle factors which may alleviate symptoms. Secondly, being aware of individuals most at risk will allow researchers to focus efforts in developing treatments more suited to high risk groups. Similarly, this will also allow governments and policy makers to target marketing campaigns to locations where prevalence of GORD is high, thereby increasing awareness of treatment options available to those with the condition.

Received: 27 March 2019; Accepted: 19 March 2020;

Published online: 02 April 2020

\section{References}

1. Nwokediuko, S. Current trends in the management of gastroesophageal reflux disease: a review. ISRN Gastroenterol. 2012, 1-11 (2012).

2. Ofman, J. The economic and quality-of-life impact of symptomatic gastroesophageal reflux disease. Am. J. Gastroenterol. 98, S8-S14 (2003).

3. Lee, Y. Y. \& McColl, K. E. Pathophysiology of gastroesophageal reflux disease. Best. Pract. Res. Clin. Gastroenterol. 27, 339-351 (2013).

4. Miller, L. et al. The esophagogastric junction. Ann. N. Y. Acad. Sci. 1232, 323-330 (2011).

5. Orlando, R. C. Overview of the mechanisms of gastroesophageal reflux. Am. J. Med. 111, 174-177 (2001).

6. Hershcovici, T., Mashimo, H. \& Fass, R. The lower esophageal sphincter. Neurogastroenterol. Motil. 23, 819-830 (2011).

7. Mason, J. \& Hungin, A. Review article: gastro-oesophageal reflux disease - the health economic implications. Aliment. Pharmacol. Ther. 22, 20-31 (2005).

8. Kubo, A. et al. Dietary guideline adherence for gastroesophageal reflux disease. BMC Gastroenterol. 14, 144 (2014).

9. Brook, R. A. et al. Cost of gastro-oesophageal reflux disease to the employer: a perspective from the United States. Aliment. Pharmacol. Ther. 26, 889-898 (2007).

10. Sandler, R. S. et al. The burden of selected digestive diseases in the United States. Gastroenterol. 22, 1500-1511 (2002).

11. Association, A. G. editor. The Burden of Gastrointestinal Diseases. Bethesda, MD: American Gastroenterological Association; (2001).

12. Moghimi-Dehkordi, B. et al. Economic burden of gastro-oesophageal reflux disease and dyspepsia: A community-based study. Arab. J. Gastroenterol. 12, 86-89 (2011).

13. Ronkainen, J. \& Agréus, L. Epidemiology of reflux symptoms and GORD. Best. Pract. Res. Clin. Gastroenterol. 27, 325-337 (2013).

14. Wong, B. \& Kinoshita, Y. Systematic review on epidemiology of gastroesophageal reflux disease in Asia. Clin. Gastroenterol. Hepatol. 4, 398-407 (2006).

15. Kinoshita, Y. et al. Systematic review of the epidemiology of gastroesophageal reflux disease in Japan. J. Gastroenterol. 46, 1092-1103 (2011).

16. Jung, H. Epidemiology of gastroesophageal reflux disease in Asia: a systematic review. J. Neurogastroenterol. Motil. 17, 14-27 (2011).

17. Delavari, A. et al. The prevalence of gastroesophageal reflux disease (GERD) in the Islamic Republic of Iran: a systematic review. Middle East. J. Dig. Dis. 4, 5-15 (2012).

18. Eusebi, L. et al. Global prevalence of, and risk factors for, gastro-oesophageal reflux symptoms: a meta-analysis. Gut. 67, 430-440 (2017).

19. Dent, J. et al. Epidemiology of gastro-oesophageal reflux disease: a systematic review. Gut. 54, 710-717 (2005).

20. El-Serag, H. et al. Update on the epidemiology of gastro-oesophageal reflux disease: a systematic review. Gut. 63, 871-880 (2013).

21. Moher, D. et al. Preferred reporting items for systematic reviews and meta-analyses: the PRISMA statement. BMJ. 339, b2535-b2535 (2009).

22. Modesti, P. et al. Panethnic differences in blood pressure in Europe: a systematic review and meta-analysis. PLoS One. 11, e0147601 (2016).

23. Schoonjans, F. MedCalc's Odds ratio calculator. MedCalc 2018. Available at, https://www.medcalc.org/calc/odds_ratio.php (2018).

24. Schoonjans F. MedCalc's Relative risk calculator. MedCalc 2018. Available at, https://www.medcalc.org/calc/relative_risk.php (2018).

25. Composition of macro Geographical (Continental) Regions, Geographical Sub-Regions, and Selected Economic and Other Groupings. Available at, http://unstats.un.org/unsd/methods/m49/m49regin.htm (2018).

26. World Population Prospects - Population Division - United Nations. Population.un.org 2018. Available at, https://population. un.org/wpp/ (2018)

27. Cai, S. et al. Overlap of gastroesophageal reflux disease and functional bowel disorders in the general Chinese rural population. J. Dig. Dis. 16, 395-399 (2015)

28. Chen, M. et al. Prevalence, risk factors and impact of gastroesophageal reflux disease symptoms: a population-based study in South China. Scand. J. Gastroenterol. 40, 759-767 (2005).

29. Cheung, T. et al. Positive association between gastro-oesophageal reflux disease and irritable bowel syndrome in a Chinese population. Aliment. Pharmacol. Ther. 25, 1099-1104 (2007).

30. Guozong, P. et al. Epidemiological study of symptomatic gastroesophageal reflux disease in China: Beijing and Shanghai. Chin. J. Dig. Dis. 1, 2-8 (2000).

31. He, J. et al. A population-based survey of the epidemiology of symptom-defined gastroesophageal reflux disease: the systematic investigation of gastrointestinal diseases in China. BMC Gastroenterol. 10 (2010).

32. Hu, W. et al. Anxiety but not depression determines health care-seeking behaviour in Chinese patients with dyspepsia and irritable bowel syndrome: a population-based study. Aliment. Pharmacol. Ther. 16, 2081-2088 (2002).

33. Ma, X. et al. Prevalence of, and factors associated with, gastroesophageal reflux disease: a population-based study in Shanghai, China. Dis. Esophagus. 22, 317-322 (2009).

34. Tan, V. et al. Gastroesophageal reflux disease: cross-sectional study demonstrating rising prevalence in a Chinese population. J. Clin. Gastroenterol. 50, e1-e7 (2016).

35. Wong, W. et al. Prevalence, clinical spectrum and health care utilization of gastro-oesophageal reflux disease in a Chinese population: a population-based study. Aliment. Pharmacol. Ther. 18, 595-604 (2003).

36. Zou, D. et al. Epidemiology of symptom-defined gastroesophageal reflux disease and reflux esophagitis: The systematic investigation of gastrointestinal diseases in China (SILC). Scand. J. Gastroenterol. 46, 133-141 (2011). 
37. Fujiwara, Y. et al. Prevalence of gastroesophageal reflux disease and gastroesophageal reflux disease symptoms in Japan. J. Gastroenterol. Hepatol. 20, 26-29 (2005).

38. Kaji, M. et al. Prevalence of overlaps between GERD, FD and IBS and impact on health-related quality of life. J. Gastroenterol. Hepatol. 25, 1151-1156 (2010).

39. Kusano, M. et al. Nationwide epidemiological study on gastroesophageal reflux disease and sleep disorders in the Japanese population. J. Gastroenterol. 43, 833-841 (2008).

40. Murao, T. et al. Lifestyle change influences on GERD in Japan: a study of participants in a health examination program. Dig. Dis. Sci. 56, 2857-2864 (2011).

41. Murase, K. et al. Gastroesophageal reflux disease symptoms and dietary behaviors are significant correlates of short sleep duration in the general population: the Nagahama study. SLEEP. 37, 1809-1815 (2014).

42. Niigaki, M. et al. Association between metabolic syndrome and prevalence of gastroesophageal reflux disease in a health screening facility in Japan. J. Gastroenterol. 48, 463-472 (2013).

43. Stanghellini, V. Three-Month prevalence rates of gastrointestinal symptoms and the influence of demographic factors: results from the domestic/international gastroenterology surveillance study (DIGEST). Scand. J. Gastroenterol. 34, 20-28 (1999).

44. Jeong, J. et al. Chronic gastrointestinal symptoms and quality of life in the Korean population. World J. Gastroenterol. 14, 6388 (2008).

45. Kang, M. et al. Abdominal obesity is an independent risk factor for erosive esophagitis in a Korean population. J. Gastroenterol. Hepatol. 22, 1656-1661 (2007).

46. Kim, J. et al. Prevalence and risk factors of Barrett’s esophagus in Korea. J. Gastroenterol. Hepatol. 22, 908-912 (2007).

47. Lee, S. et al. Prevalence of endoscopic reflux esophagitis among Koreans. J. Gastroenterol. Hepatol. 16, 373-376 (2001)

48. Lee, S. et al. Prevalence and risk factors for overlaps between gastroesophageal reflux disease, dyspepsia, and irritable bowel syndrome: a population-based study. Digestion. 79, 196-201 (2009).

49. Min, B. et al. Prevalence of uninvestigated dyspepsia and gastroesophageal reflux disease in korea: a population-based study using the rome iii criteria. Dig. Dis. Sci. 59, 2721-2729 (2014).

50. Yang, S. et al. Prevalence of gastroesophageal reflux disease symptoms and uninvestigated dyspepsia in Korea: a population-based study. Dig. Dis. Sci. 53, 188-193 (2008).

51. Hung, L. et al. Prevalence of gastroesophageal reflux disease in a general population in Taiwan. J. Gastroenterol. Hepatol. 26, 1164-1168 (2011).

52. Abdullah, M. et al. Prevalence, risk factors and socio-epidemiological study of gastroesophageal reflux disease: an urban population based study in Indonesia. Asian J. Epidemiol. 9, 18-23 (2016).

53. Shaha, M. et al. Prevalence and risk factors for gastro-esophageal reflux disease in the North-Eastern part of Bangladesh. Bangladesh Med. Res. Counc. Bull. 38, 108-113 (2013).

54. Bhatia, S. et al. Epidemiology and symptom profile of gastroesophageal reflux in the Indian population: Report of the Indian Society of Gastroenterology Task Force. Indian. J. Gastroenterol. 30, 118-127 (2011).

55. Kumar, S. \& Shivalli, S. Prevalence, perceptions and profile of gastroesophageal reflux disease in a rural population of North Bihar. Nat. J. Commun. Med. 5, 214-218 (2014).

56. Wang, H. et al. Prevalence of gastro-esophageal reflux disease and its risk factors in a community-based population in southern India. BMC Gastroenterol 16 (2016).

57. Aletaha, N. et al. The role of psychosocial disorders in gastroesophageal reflux disease. Govaresh. 12, 92-97 (2007).

58. Darvishmoghadam, S. et al. Review of clinical spectrum of gastroesophageal reflux disease in a general population; a study from south-east Iran. Middle East. J. Dig. Dis. 8, 310-317 (2016).

59. Ehsani, M. et al. Epidemiology of gastroesophageal reflux disease in Tehran, Iran. J. Gastroenterol. Hepatol. 22, 1419-1422 (2007).

60. Islami, F. et al. Determinants of gastroesophageal reflux disease, including hookah smoking and opium use- a cross-sectional analysis of 50,000 individuals. PLoS One. 9, e89256 (2014)

61. Khodamoradi, Z. et al. Prevalence and correlates of gastroesophageal reflux disease in southern Iran: Pars cohort study. Middle East. J. Dig. Dis. 9, 129-138 (2017).

62. Mansour-Ghanae, F. et al. The epidemiology of gastroesophageal reflux disease: a survey on the prevalence and the associated factors in a random sample of the general population in the Northern part of Iran. Int. J. Mol. Epidemiol. Genet. 4, 175-182 (2013).

63. Mostaghni, A. et al. Prevalence and risk factors of gastroesophageal reflux disease in Qashqai migrating nomads, southern Iran. World J. Gastroenterol. 15, 961 (2009).

64. Nasseri-Moghaddam, S. et al. Epidemiological study of gastro-oesophageal reflux disease: reflux in spouse as a risk factor. Aliment. Pharmacol. Ther. 28, 144-153 (2008).

65. Nouraie, M. et al. Epidemiology of gastroesophageal reflux symptoms in Tehran, Iran: a population-based telephone survey. Arch. Iran. Med. 10, 289-294 (2007).

66. Nouraie, M. et al. Hygiene could affect GERD Prevalence independently: a population-based study in Tehran. Am. J. Gastroenterol. 102, 1353-1360 (2007).

67. Pourhoseingholi, A. et al. Epidemiological features of gastro-esophageal reflux disease in Iran based on general population. Gastroenterol. Hepatol. Bed Bench. 5, 54-59 (2012).

68. Rogha, M. et al. Gastroesophageal reflux disease in Esfahan. Govaresh. 11, 145-149 (2006).

69. Saberi-Firoozi, M. et al. Risk factors of gastroesophageal reflux disease in Shiraz, southern Iran. World J. Gastroenterol. 13, 5486 (2007).

70. Solhpour, A. et al. Gastro-esophageal reflux aymptoms and body mass index: no relation among the Iranian population. Indian. J. Gastroenterol. 27, 153-155 (2008).

71. Vossoughinia, H. et al. An epidemiological study of gastroesophageal reflux disease and related risk factors in urban population of Mashhad, Iran. Iran Red Crescent Med J. 16 (2014).

72. Yadegarfar, G. et al. Iranian lifestyle factors affecting reflux disease among healthy people in Qom. Electron. Physician. 10, 6718-6724 (2018)

73. Bor, S., Kitapcioglu, G. \& Kasap, E. Prevalence of gastroesophageal reflux disease in a country with a high occurrence of Helicobacter pylori. World J. Gastroenterol. 23, 525 (2017).

74. Kitapcioglu, G. et al. Overlap of symptoms of dyspepsia and gastroesophageal reflux in the community. Turk. J. Gastroenterol. 18, 14-19 (2007).

75. Mungan, Z. Prevalence and demographic determinants of gastroesophageal reflux disease (GERD) in the Turkish general population: A population-based cross-sectional study. Turk. J. Gastroenterol. 23, 323-332 (2012).

76. Yonem, O. et al. Gastroesophageal reflux disease prevalence in the city of Sivas. Turk. J. Gastroenterol. 24, 303-310 (2013).

77. Moshkowitz, M. Gastroesophageal reflux disease symptoms: Prevalence, sociodemographics and treatment patterns in the adult Israeli population. World J. Gastroenterol. 17, 1332 (2011).

78. Sperber, A. et al. Prevalence of GERD symptoms in a representative Israeli adult population. J. Clin. Gastroenterol. 41, 457-461 (2007).

79. Almadi, M. et al. Prevalence of symptoms of gastroesopahgeal reflux in a cohort of Saudi Arabians: A study of 1265 subjects. Saudi J. Gastroenterol. 20, 248 (2014).

80. Alsuwat, O. et al. Prevalence of gastroesophageal reflux disease in Saudi Arabia. J. Clin. Med. Res. 10, 221-225 (2018). 
81. Ziółkowski, B. et al. Prevalence of abdominal symptoms in the Polish population. Prz. Gastroenterol. 1, 20-25 (2012).

82. Chirila, I. et al. The role of diet in the overlap between gastroesophageal reflux disease and functional dyspepsia. Turk. J. Gastroenterol. 27, 73-80 (2016).

83. Bor, S. et al. Prevalence of gastroesophageal reflux disease in Moscow. Dis. Esophagus. 29, 159-165 (2016).

84. Lazebnik, L. B. et al. Multicentre study "Epidemiology of gastroesophageal reflux disease in Russia" (MEGRE): first results. Clin Exp Gastroenterol. 4-12 (2009).

85. Çela, L. et al. Lifestyle characteristics and gastroesophageal reflux disease: a population-based study in Albania. Gastroenterol. Res. Pract. 2013, 1-7 (2013).

86. Zagari, R. et al. Gastro-oesophageal reflux symptoms, oesophagitis and Barrett's oesophagus in the general population: the LoianoMonghidoro study. Gut. 57, 1354-1359 (2008).

87. Papatheodoridis, G. \& Karamanolis, D. Prevalence and impact of upper and lower gastrointestinal symptoms in the Greek Urban general population. Scand. J. Gastroenterol. 40, 412-421 (2005).

88. Diaz-Rubio, M. et al. Symptoms of gastro-oesophageal reflux: prevalence, severity, duration and associated factors in a Spanish population. Aliment. Pharmacol. Ther. 19, 95-105 (2004).

89. Ponce, J. et al. Prevalence of gastro-oesophageal reflux disease in Spain and associated factors. Aliment. Pharmacol. Ther. 23, 175-184 (2006).

90. Rey, E. et al. Onset and disappearance rates of gastroesophageal reflux symptoms in the Spanish population, and their impact on quality of life. Rev Esp Enferm Dig. 101 (2009).

91. Schwenkglenks, M., Marbet, U. \& Szucs, T. Epidemiology and costs of gastroesophageal reflux disease in Switzerland: a populationbased study. Sozial-Und Preventivmedizin/Social Preventive Medicine. 49, 51-61 (2004).

92. Bretagne, J. F. et al. Le reflux gastro-œesophagien dans la population générale française: résultats d'une enquête sur 8000 sujets adultes. La. Presse Médicale. 35, 23-31 (2006).

93. Des Varannes, S. B. et al. Gastroesophageal reflux disease in primary care. Gastroenterol. Clin. Biol. 30, 364-370 (2006).

94. Bollschweiler, E. et al. Prevalence of dysphagia in patients with gastroesophageal reflux in Germany. Dysphagia. 23, 172-176 (2008).

95. Djärv, T. Physical activity, obesity and gastroesophageal reflux disease in the general population. World J. Gastroenterol. 18, 3710 (2012).

96. Ronkainen, J. et al. High prevalence of gastroesophageal reflux symptoms and esophagitis with or without symptoms in the general adult Swedish population: a Kalixanda study report. Scand. J. Gastroenterol. 40, 275-285 (2005).

97. Terry, P. et al. Reflux-inducing dietary factors and risk of adenocarcinoma of the esophagus and gastric cardia. Nutr. Cancer. 38, 186-191 (2000).

98. Wiklund, I., Carlsson, J. \& Vakil, N. Gastroesophageal reflux symptoms and well-being in a random sample of the general population of a Swedish community. Am. J. Gastroenterol. 101, 18-28 (2006).

99. Isolauri, J. \& Laippala, P. Prevalence of Symptoms Suggestive of gastroesophageal reflux disease in an adult population. Ann. Med. 27, 67-70 (1995).

100. Ness-Jensen, E. et al. Changes in prevalence, incidence and spontaneous loss of gastro-oesophageal reflux symptoms: a prospective population-based cohort study, the HUNT study. Gut. 61, 1390-1397 (2012).

101. Mohammed, I. Genetic influences in gastro-oesophageal reflux disease: a twin study. Gut. 52, 1085-1089 (2003).

102. Mohammed, I., Nightingale, P. \& Trudgill, N. Risk factors for gastro-oesophageal reflux disease symptoms: a community study. Aliment. Pharmacol. Ther. 21, 821-827 (2005).

103. Murray, L. et al. Relationship between body mass and gastro-oesophageal reflux symptoms: The Bristol Helicobacter Project. Int. J. Epidemiol. 32, 645-650 (2003).

104. Penston, J. \& Pounder, R. A survey of dyspepsia in Great Britain. Aliment. Pharmacol. Ther. 10, 83-89 (2007).

105. Rasmussen, S. et al. Overlap of symptoms of gastroesophageal reflux disease, dyspepsia and irritable bowel syndrome in the general population. Scand. J. Gastroenterol. 50, 162-169 (2015).

106. Crews, N. et al. Prevalence and predictors of gastroesophageal reflux complications in community subjects. Dig. Dis. Sci. 61, 3221-3228 (2016).

107. Dean, B. et al. The relationship between the prevalence of nighttime gastroesophageal reflux disease and disease severity. Dig. Dis. Sci. 55, 952-959 (2010).

108. Farup, C. et al. The impact of nocturnal symptoms associated with gastroesophageal reflux disease on health-related quality of life. Arch. Intern. Med. 161, 45 (2001).

109. Jung, H. et al. Overlap of gastro-oesophageal reflux disease and irritable bowel syndrome: prevalence and risk factors in the general population. Aliment. Pharmacol. Ther. 26, 453-461 (2007).

110. Locke, G. et al. Prevalence and clinical spectrum of gastroesophageal reflux: A population-based study in Olmsted County, Minnesota. Gastroenterol. 112, 1448-1456 (1997).

111. Locke, G. et al. Risk factors associated with symptoms of gastroesophageal reflux. Am. J. Med. 106, 642-649 (1999).

112. Yuen, E. et al. Prevalence, knowledge and care patterns for gastro-oesophageal reflux disease in United States minority populations. Aliment. Pharmacol. Ther. 32, 645-654 (2010).

113. Chiocca, J. et al. Prevalence, clinical spectrum and atypical symptoms of gastro-oesophageal reflux in Argentina: a nationwide population-based study. Aliment. Pharmacol. Ther. 22, 331-342 (2005).

114. Oliveira, S. S. D. et al. Gastroesophageal reflux disease: prevalence and associated factors. Arq. Gastroenterol. 42, 116-121 (2005).

115. Dacoll, C. et al. Prevalence of gastroesophageal reflux disease in Uruguay. Gastroenterol. Hepatol. 35, 460-467 (2012).

116. Páramo-Hernández, D. B. et al. Prevalence of gastro-esophageal reflux symptoms and associated factors: a population survey in the principal cities of Colombia. Rev. Colomb. Gastroenterol. 31, 337-346 (2016).

117. Eslick, G. \& Talley, N. Gastroesophageal reflux disease (GERD): risk factors, and impact on quality of life-a population-based study. J. Clin. Gastroenterol. 43, 111-117 (2009).

118. Pandeya, N., Green, A. \& Whiteman, D. Prevalence and determinants of frequent gastroesophageal reflux symptoms in the Australian community. Dis. Esophagus. 25, 573-583 (2012)

119. Talley, N., Boyce, P. \& Jones, M. Identification of distinct upper and lower gastrointestinal symptom groupings in an urban population. Gut. 42, 690-695 (1998).

120. Haque, M. et al. Prevalence, severity and associated features of gastro-oesophageal reflux and dyspepsia: a population-based study. N. Z. Med. J. 113, 178 (2000).

121. Nwokediuko, S. Gastroesophageal reflux disease: a population based study. Gastroenterol. Res. 2, 152 (2009).

122. Lohouès-Kouacou, M. J. et al. Prévalence du reflux gastro-œsophagien typique à Abidjan Prevalence of typical gastro-esophageal reflux in Abidjan. J. Africain d'Hépato-Gastroentérol. 7, 117-121 (2013).

123. Ng, M. et al. Global, regional, and national prevalence of overweight and obesity in children and adults during 1980-2013: a systematic analysis for the Global Burden of Disease Study 2013. Lancet. 384, 766-781 (2014).

124. Division U. UNSD - Demographic and Social Statistics. Unstats.un.org 2018. Available at, https://unstats.un.org/unsd/ demographic-social/sconcerns/mar_divorce/ (2018)

125. Mungan, Z. \& Pinarbasi Simsek, B. Which drugs are risk factors for the development of gastroesophageal reflux disease? Turk. J. Gastroenterol. 28, 38-43 (2017). 
126. McCarthy, D. Prevention and treatment of gastrointestinal symptoms and complications due to NSAIDs. Best. Pract. Res. Clin. Gastroenterol. 15, 755-773 (2001).

127. Kulkarni, S. Gastric emptying of solids in long-term NSAID users: correlation with endoscopic findings and Helicobacter pylori status. Am. J. Gastroenterol. 94, 382-386 (1999).

128. Horowitz, M. et al. The effect of chilli on gastrointestinal transit. J. Gastroenterol. Hepatol. 7, 52-56 (1992).

129. Milke, P. et al. Gastroesophageal reflux in healthy subjects induced by two different species of chilli (Capsicum annum). Dig. Dis. 24, 184-188 (2006).

130. Vakil, N. et al. The Montreal definition and classification of gastroesophageal reflux disease: a global evidence-based consensus. Am. J. Gastroenterol. 101, 1900-1920 (2006).

\section{Acknowledgements}

The authors acknowledge the financial support provided by the University of Huddersfield, Huddersfield, UK.

\section{Author contributions}

Jorabar Singh Nirwan: acquisition of data, analysis, interpretation of data, statistical analysis, methodology, writing-original draft and writing- review and editing, Syed Shahzad Hasan: analysis, interpretation of data, statistical analysis, methodology and writing- review and editing, Zaheer-Ud-Din Babar: writing- review and editing, Barbara R. Conway: methodology and writing- review and editing and Muhammad Usman Ghori: study concept and design, acquisition of data, analysis and interpretation of data, statistical analysis, methodology and writing- review and editing, Project administration and supervision.

\section{Competing interests}

The authors declare no competing interests.

\section{Additional information}

Supplementary information is available for this paper at https://doi.org/10.1038/s41598-020-62795-1.

Correspondence and requests for materials should be addressed to M.U.G.

Reprints and permissions information is available at www.nature.com/reprints.

Publisher's note Springer Nature remains neutral with regard to jurisdictional claims in published maps and institutional affiliations.

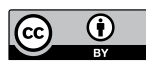

Open Access This article is licensed under a Creative Commons Attribution 4.0 International License, which permits use, sharing, adaptation, distribution and reproduction in any medium or format, as long as you give appropriate credit to the original author(s) and the source, provide a link to the Creative Commons license, and indicate if changes were made. The images or other third party material in this article are included in the article's Creative Commons license, unless indicated otherwise in a credit line to the material. If material is not included in the article's Creative Commons license and your intended use is not permitted by statutory regulation or exceeds the permitted use, you will need to obtain permission directly from the copyright holder. To view a copy of this license, visit http://creativecommons.org/licenses/by/4.0/.

(c) The Author(s) 2020 